\title{
Divide and Inform: \\ Rationing Information to Facilitate Persuasion
}

\author{
Beatrice Michaeli
}

Submitted in partial fulfillment of the requirements for the degree of Doctor of Philosophy under the Executive Committee of the Graduate School of Arts and Sciences

\section{COLUMBIA UNIVERSITY}


Beatrice Michaeli

All rights reserved 


\section{ABSTRACT \\ Divide and Inform: Rationing Information to Facilitate Persuasion Beatrice Michaeli}

This paper develops a Bayesian persuasion model examining a manager's incentives to gather information when the manager can disseminate this information selectively to users and when the objectives of the manager and the users are not perfectly aligned. The model predicts that, if the manager can choose the subset of users to receive the information, then the manager may gather more precise information. The paper identifies conditions under which a regime that allows managers to grant access to information selectively maximizes aggregate information. Strikingly, this happens when the objectives of managers and users are sufficiently misaligned. These results call into doubt the common belief that forcing managers to provide unrestricted access to information to all potential users is always beneficial. 


\section{Contents}

1 Introduction 1

2 Model Setup 9

3 Access to Information 13

4 Costly Persuasion $\quad 22$

4.1 The Optimal Precision . . . . . . . . . . . . . . 25

4.2 Regime Preferences . . . . . . . . . . . . . . . . . . 28

4.3 Welfare Analysis with Sufficiently Aligned Preferences . . . . . . . . 30

4.4 Regulated Information Dissemination . . . . . . . . . . . . . 33

5 Biased Users of Information 35

6 Concluding Remarks $\quad 40$

$\begin{array}{ll}\text { References } & 42\end{array}$

$\begin{array}{ll}\text { Appendix } & 46\end{array}$ 


\section{ACKNOWLEDGMENTS}

I would like to thank Philip Berger, Hans Christensen, Wouter Dessein, Fabrizio

Ferry, Paul Fischer, Henry Friedman, John Hughes, Pingyang Gao, Jonathan Glover, Tim Gray, Ilan Guttman, Mirko Heinle, Moritz Hiemann, Emir Kamenica, Christian Leuz, Mark Maffett, Haresh Sapra, Catherine Schrand, Brett Trueman and seminar participants at Booth School of Business, Carnegie Melon University, Columbia Business School, Kellog School of Management, Tel Aviv University, Tuck School of Business, UC Berkeley, UCLA, Wharton and Yale School of Management for their helpful comments and suggestions. I am grateful to Nahum Melumad, Xiaojing Meng and Amir Ziv for their valuable discussions that benefited this project. I am very indebted to Navin Kartik for his excellent guidance and motivation. My deepest gratitude goes to Tim Baldenius not only for the continuous support of my PhD study and guidance in research, but also for his endless enthusiasm, inspiration and encouragement that made the PhD program an unforgettable journey. 


\section{Introduction}

Common practices, rules and regulations require that managers communicate information to all potential internal and external users of this information. Regulation Fair Disclosure (Reg FD), for example, prohibits selective disclosure. Broadly speaking, any such regulations pursue the dual objectives (i) to level the playing field, i.e., ensure fairness, and (ii) to increase the aggregate amount of information in the marketplace: "the market is best served by more, not less, disclosure of information by issuers." ${ }^{1}$ While the fairness benefits of Reg FD appear uncontroversial, the informational benefits are less so. One might reason that, after Reg FD, the consensus analyst forecast would be more accurate because all analysts receive the same access to information. The literature, however, provides mixed empirical evidence. Herrmann, Hope and Thomas (2008), for example, show an increase in the average analyst forecast accuracy, while Agrawal, Chadha and Chen (2006) document a decrease. Francis, Nanda and Wang (2006) and Heflin, Subramanyam and Zhang (2003) find no evidence of significant change. Moreover, at a post-adoption roundtable discussion, analysts and other panelists expressed concerns that Reg FD had diminished the quantity and quality of the information disseminated by firms. ${ }^{2}$ Illuminating the mechanisms that drive these observations requires a theoretical framework that links the managers' ability to disseminate information selectively with factors that influence the quality of information.

The standard view is fairness and greater availability of information at an aggregate level go "hand in hand". The justification for this view is that, provided

\footnotetext{
${ }^{1}$ See Selective Disclosure and Insider Trading, SEC Release Nos. 33-7881, 34-43154, IC-24599, File No. S7-31-99 (Aug. 15 2000), http://www.sec.gov/rules/final/33-7881.htm.

${ }^{2}$ See Regulation Fair Disclosure Revisited, Report by Commissioner Laura S. Unger, http://www.sec.gov/news/studies/regfdstudy.htm.
} 
managers report truthfully, they disseminate the same quality of information regardless of who observes it. However, this argument ignores the fact that the quality of information is endogenous. Managers choose not only to whom to disclose the information but also whether and what quality of information to gather in the first place, and this choice may be affected by the size of the anticipated audience.

Managers' incentives to gather information also depend on their objectives. Often their objectives are not perfectly aligned with those of users. Consider, as an example, information dissemination by a manager to financial analysts (hereafter, "the leading example"). Analysts interact with investors repeatedly and therefore have long-term incentives to provide accurate forecasts. In addition, as Groysberg, Healy, and Maber (2011) show, part of analysts' compensation is based on trading commissions. Thus, to amplify trading volume, analysts may have short-term incentives to bias their forecasts upward in good states and downwards in bad ones (Beyer and Guttman, 2011). The firm manager, on the other hand, may prefer a consensus forecast that only partially reflects the economic earnings and is biased toward a specific value. For example, the manager may be biased toward a high forecast because it increases the firm's stock price and hence her stock-based compensation. ${ }^{3}$

This paper studies the effects of managers' discretion to limit access to information to a subset of users on the managers' incentives to gather information and thereby on the aggregate information available to market participants (which, within the confines of the model, allows me to make inferences about ex ante effi-

\footnotetext{
${ }^{3}$ The standard view is that managers like high forecasts, but this need not always be the case. For example, managers may like low forecasts before option grant date to trigger a low exercise price. In addition, low forecasts are easier to beat.
} 
ciency). In doing so, it examines whether there is a trade-off between the fairness and information objectives mentioned above. I develop a model of Bayesian persuasion with information control that builds on Kamenica and Gentzkow (2011). ${ }^{4}$ The players - a firm manager ("she") and a group of identical users ("they" in plural, "he" in singular) - have misaligned preferences. The manager can influence the sensitivity with which the users will react to the information eventually revealed by choosing the precision of an information system that she implements. The main innovation of my model is that the manager's preferences over the actions of a certain subset of users depend on the actions of the users outside this subset. ${ }^{5}$ As a result, besides controlling the information precision, the manager, if granted discretion, can also control the aggregate amount of information by strategically choosing the subset of users who observe the signal of the state of nature (e.g., the economic earnings).

The first part of the paper considers a stylized setting in which the implementation of an information system is cost-free. The analysis shows that, if the manager does not have discretion over the access to information, she implements a perfectly revealing information system only if her preferences are sufficiently aligned with those of the users. $^{6}$ The intuition behind this result is that, when the players' objectives are sufficiently misaligned, the users react too sensitively to the signal from the manager's point of view. Hence the manager is better off not providing

\footnotetext{
${ }^{4}$ In the leading example, shifting the focus to the information gathering stage links two issues that are commonly considered by separate research strands: (i) internal information system implementation and control (usually analyzed in managerial accounting) and (ii) information communication to external users (usually analyzed in financial accounting).

${ }^{5}$ In the leading example, managers care about the consensus forecast, and so their preferences over the forecasts of a certain subset of analysts depend on the forecasts of the other analysts.

${ }^{6}$ This is a variation of the findings in Kamenica and Gentzkow (2011).
} 
information and leaving the users act on their prior. ${ }^{7}$ In contrast, if the manager has discretion over restricting access to information, she always implements a perfectly revealing system. This result is not immediately intuitive for a setting with ex ante identical users. The rationale behind it is that by keeping some of the users "in the dark" the manager can regulate the sensitivity of the users' aggregate action to changes in the state of nature. As a result of this additional degree of freedom, the manager now finds it in her best interest to implement the most informative system, the output of which is only selectively disseminated. As one would expect, the optimal fraction of privileged users who get to observe the signal is increasing in the degree of players' objective alignment.

A corollary of the preceding discussion is that, if the players' preferences are sufficiently misaligned, a regime that allows for information discrimination ("unregulated dissemination") maximizes aggregate information. Among the players considered, unregulated dissemination Pareto dominates a regime that requires information dissemination to all users ("mandated dissemination"). It makes better off the manager (by revealed preference) as well as some users (because they receive information that would not be available otherwise) without making the other users worse off (because they do not observe information anyway). ${ }^{8}$ Paradoxically, when the players' preferences over actions are misaligned, their preferences over regimes are aligned. The opposite is also true: when the players' preferences over actions

\footnotetext{
${ }^{7}$ As anecdotal evidence, in July 2013 SEC required Urban Outfitters to publicly disclose the effect of direct-to-customer sales on the net retail segment sales. In response, Urban Outfitters declared that effective Q1/2014 the company will no longer gather this information even for internal purposes.

${ }^{8}$ To focus on the information gathering incentives of the manager I do not model a trading game. Asymmetric dissemination in a larger model with investors "buying " information from analysts and then trading on this information, would redistribute (expected) trading surplus and will leave those investors not in the loop worse off. No Pareto ranking then could be made.
} 
are sufficiently aligned, their preferences over regimes are misaligned. The manager then prefers unregulated dissemination, while all users at least weakly prefer mandated dissemination. This result calls into doubt the conventional wisdom that regulating information dissemination and requiring equal access to information is especially needed when the incentive conflict is severe. Ironically, under this scenario, regulations forcing equal access to information will promote fairness but at the expense of reduced overall information.

With costless information acquisition, the optimal precision is a bang-bang solution: the manager implements either a perfectly revealing information system or one that does not convey any information at all. However, interior precision levels and information acquisition costs are frequently observed in practice. Therefore, in the second part of the paper, I consider costly implementation. I find that, if the fraction of informed users were exogenous, then the interior optimal precision level would be lower, the farther this fraction is from the one the manager would choose if she could.

When the preferences between the players are misaligned, the Pareto ranking of mandated and unregulated dissemination regimes remains the same. However, when preferences over actions are aligned, the introduction of precision costs creates disagreement between the users regarding the preferred regime. The assessment of the aggregate users' welfare depends on two countervailing effects: (i) a precision effect - the information collected under unregulated dissemination is more precise than under mandated dissemination and (ii) an omission effect - the fraction of users who observe the information under unregulated dissemination is lower than under mandated dissemination. The paper identifies sufficient conditions under which the users are better off, on an aggregate level, under unregulated dissem- 
ination. Put differently, even the users themselves, at some prior state, would collectively agree to unregulated dissemination, as long as they are behind the veil of ignorance (Harsanyi, 1955), i.e., before each learns whether he will be included in the group of informed users.

Another way to look at the efficiency effects of the manager's ability to limit access to information is by considering the accuracy of the aggregate users' action. In the leading example, this reflects the accuracy of the analysts' consensus forecast. My results suggests that at least some of the inconclusive findings in the empirical literature on the changes in forecast accuracy before and after Reg FD may be due to unobserved cross-sectional differences, e.g., in the degree of interest alignment between management and analysts or the costs associated with the implementation of internal controls.

My model has features in common with the mandatory and the voluntary disclosure literature. The key difference is the timing: the information dissemination is only voluntary ex ante when the manager decides whether to implement an information system. However, once an implemented system has generated a signal, the manager must truthfully share it with the predetermined group of users. The assumption of truthful reporting can be motivated in several ways. First, the manager may not be willing to bias the signal due to personal integrity or the threat of litigation. The stringent rules for accountability and transparency after Dodd Frank and the stronger internal control requirements of Sarbanes-Oxley Section 404 leave less reporting discretion to managers. Second, in many cases, the signal is verifiable. Lastly, the state of nature can be interpreted as the most informative signal the manager can generate. Her choice of signal precision is then equivalent to the choice of how much to garble information. 
This paper belongs to the persuasion literature. Its primary theoretical antecedent is the Bayesian persuasion model of Kamenica and Gentzkow (2011). As in Alonso and Camara (2014) and Wang (2013), this paper focuses on information control with multiple receivers. Similar to Gentzkow and Kamenica (2013), the model allows for persuasion to be costly.

A key application of my study is financial reporting to external users. Broadly speaking, my paper is related to the literature on costs and benefits of information dissemination, which is reviewed by Verrecchia (2001), Dye (2001), Beyer, Cohen, Lys, and Walther (2010) and Stocken (2012). Prior literature on selective disclosure considers models that focus on the ex post strategic communication between players with asymmetric information (e.g., cheap-talk and disclosure models). I assume that the players have symmetric information and focus on the ex ante strategic control of the information environment at the information gathering stage. This allows for identification of unintended consequences of regulations advocating comprehensive information dissemination, e.g., Reg FD, which are qualitatively different from the already studied effects driven by herding (e.g., Arya, Glover, Mittendorf and Narayanamoorthy, 2005), externalities (e.g., Chen, Lewis and Zhang, 2013), users' incentives to gather information (e.g., Jorgensen, Li and Melumad, 2013; Demski and Feltham, 1994; Kim and Verrecchia, 1991; McNichols and Trueman, 1994), price efficiency (Dutta, 1996) and private information sale (Bushman, 1991 and Sabino, 1993). Studies that are more closely related to mine are Edmans, Heinle and Huang (2013) and Gao and Liang (2013) because they consider ex ante commitment to dissemination policy. Edmans, Heinle and Huang (2013) consider real effects and find that the optimal level of disclosure is a trade-off between the benefits of disclosure (reduced cost of capital) and its costs (inefficient investment). 
In Gao and Liang (2013) the firm's information precision is exogenous, and the focus is on the informational feedback effect: a speculator can privately acquire information that, once reflected in the stock price, can be used by the firm when choosing an investment.

My paper is related to the literature that links ex ante information acquisition and ex post communication strategic choices (e.g., Che and Kartik, 2009; Fischer and Stocken, 2010; Hughes and Pae, 2004; Pae, 1999). In these studies, a player (e.g., advisor, analyst, entrepreneur) acquires information and then strategically decides whether or how to communicate it to another player. Shavell (1994) compares a seller's incentives to acquire information under mandatory and voluntary disclosure to buyers.

In my model, once the output of the information system is generated, the manager must truthfully communicate it to the users. Hence my paper relates to the literature that considers the firm's choice of report precision that is publicly disclosed to investors (e.g., Penno, 1996; Titman and Trueman, 1986). In my study, the choice of information system is a strategic persuasion device for control of information environment. In that sense, my paper also relates to the strand of literature studying information system design as a commitment device (e.g., Baiman, 1975; Arya, Glover and Sivaramakrishnan, 1997).

The paper proceeds as follows. Section 2 describes the model. Section 3 analyzes the access to information and considers policy implications. Section 4 extends the results to a setting in which persuasion is costly and discusses the socially optimal fraction of informed users. Section 5 considers users' bias. Section 6 concludes. All proofs are in the appendix. 


\section{Model Setup}

I consider a firm manager and a group of individuals (labeled "users") who are interested in learning about the firm. There is a continuum of identical users uniformly distributed on the interval $[0,1]$. The users simultaneously take actions. The payoff of user $i$ depends on his own action $a_{i} \in \mathbb{R}$ and the state of nature of the firm $\omega \in \Omega \in \mathbb{R}$ (for example, economic earnings):

$$
u\left(a_{i}, \omega\right)=-\left(a_{i}-\omega\right)^{2}
$$

where $\Omega$ is a finite set. In the leading example, $a_{i}$ represents the individual analyst's forecast. For any realization of the state of nature, the interior solution that maximizes the payoff of user $i$ is

$$
a^{*}(\omega) \in \arg \max _{a_{i}} u\left(a_{i}, \omega\right)=\omega
$$

i.e., a representative user prefers an action that is fully aligned with the state of nature. In the leading example from the introduction this assumption reflects the fact that analysts care about their reputation for accuracy. ${ }^{9}$

The payoff of the manager depends on the aggregate action of all users:

$$
A \equiv \int_{0}^{1} a_{i} d i
$$

\footnotetext{
${ }^{9}$ Analysts might be upward biased in good states and downward biased in bad states to amplify the trading volume. Allowing for different sensitivity to the state of nature of the users' bliss point will not change the results qualitatively as long as the users' preferred actions are more sensitive to the state of nature than the manager's preferred aggregate action.
} 
and on the state of nature $\omega$ and is denoted by:

$$
v(A, \omega)=-(A-k \omega-(1-k) \bar{\omega})^{2},
$$

where $k \in(0,1)$ and $\bar{\omega} \in \mathbb{R}$ are parameters. The assumption that the manager's payoff is affected by the aggregate actions of all users is a key feature of the model and is motivated by the fact that in many cases the users are atomistic, i.e., the actions of a single user do not affect the firm's wealth. In the leading example, $A$ represents the consensus analysts' forecast. For any realization of the state of nature, the interior solution that maximizes the manager's payoff is

$$
A^{*}(\omega) \in \arg \max _{A} v(A, \omega)=k \omega+(1-k) \bar{\omega}
$$

The manager prefers an aggregate action that is partially aligned with the state of nature $\omega$ and partially biased toward some exogenous value $\bar{\omega}{ }^{10}$ In the leading example, this assumption reflects the fact that the manager may want a consensus forecast that is less sensitive to the economic earnings and is biased toward a specific forecast that maximizes the manager's compensation. ${ }^{11}$ I refer to $k$ as the

\footnotetext{
${ }^{10}$ A similar preference was introduced in a cheap talk setting by Melumad and Shibano (1991) and by Kamenica and Gentzkow (2011) in their lobbying example of a Bayesian persuasion game. In Melumad and Shibano (1991), the communication game is affected by preference reversal, i.e., the bliss point of the manager can be lower or higher than the bliss point of the user in different environments. In my model, although preference reversal is possible depending on the relative magnitude of $\bar{\omega}$ vis-a-vis $\omega$, it does not affect the persuasion game. As I show, by implementing an information system and limiting access to information, the manager can persuade the users to take an aggregate action that is closer to $k \omega$ but cannot persuade them to take an action closer to $(1-k) \bar{\omega}$.

${ }^{11}$ The standard view would be that this preferred forecast is high so that it increases the stock price and the manager's stock-based compensation. However, it could also be a low forecast (to trigger low exercise price before option grant date) or a mean forecast (that is easy to meet or beat).
} 
measure of "preference alignment" between the manager and a representative user. As $k \rightarrow 1$, the players' preferences are perfectly aligned because then the manager, just like the users, prefers an action that is fully aligned with the state of nature.

None of the players observes the state of nature $\omega$. I assume that $\omega$ is distributed according to some (commonly known) probability distribution $\pi_{o} \in \Delta(\Omega)$ with mean $\mu_{0} \equiv E[\omega]$ and variance $\operatorname{Var}(\omega) .{ }^{12}$ The manager can implement an information system that will provide a noisy signal $s \in \Omega \in \mathbb{R}$ of the state of nature. ${ }^{13}$ In the basic setting, the information system implementation is cost-free. This assumption is relaxed in Section 4. Each signal realization leads to a posterior belief $B \in \Delta(\Omega)$. An information system creates a (commonly known) distribution over posterior beliefs $\pi \in \Delta(\Delta(\Omega))$. Throughout the paper I refer to $\pi$ as the design of the information system. Let $\operatorname{supp}(\pi) \subseteq \Delta(\Omega)$ be the set of all possible posteriors. The distribution of posteriors $\pi$ has to be Bayes-plausible, i.e.,

$$
\pi_{0}=\int B \pi(d B)
$$

Let $\Pi\left(\pi_{0}\right)$ denote the set of all Bayes-plausible information systems. Then,

$$
\Pi\left(\pi_{0}\right)=\left\{\pi \in \Delta(\Delta(\Omega)): \pi_{0}=\int B \pi(d B)\right\}
$$

Note that $\Pi\left(\pi_{0}\right)$ includes an information system with $s=\omega$, i.e., such that perfectly reveals the state of nature. For the reminder of the paper I will refer to such system as "fully revealing" or "perfectly informative".

\footnotetext{
${ }^{12}$ This rules out distributions with undefined mean and variance, e.g., Cauchy distribution.

${ }^{13}$ For example, managers hire economists to provide forecasts, purchase inventory management software, pay for appraisals to assess the value of the firm's long-lived assets, etc.
} 
To analyze the effects of the ability (or lack thereof) of the manager to limit access to information, I compare two alternative regimes: "mandatory dissemination" (hereafter, "MD") and "unregulated dissemination" (hereafter, "UD"). Under MD the manager is required to disclose the same signal to all users, while under UD the manager can choose the fraction $x \in[0,1]$ of users who will observe the signal. ${ }^{14} \mathrm{I}$ refer to those users as "informed" and to the rest of the users as "uninformed." In other words, ex ante identical users become heterogeneous endogenously by virtue of selective information dissemination. Observability of $x$ is irrelevant for the analysis because, by assumption, each user only cares about his own action.

I restrict attention to cases in which the signal is truthfully communicated to the informed users. This assumption reflects the litigation threat in case of concealing or distorting information. In my model, the users do not gather information on their own. This assumption is motivated by the fact that, in many cases, firm managers have access to sources of information unavailable to the users. In the leading example, only the firm manager (and not the analyst) can gather information about within-firm determinants of economic earnings, e.g., production capacity and inventory levels. ${ }^{15}$ Furthermore, the users cannot contract on the information system design because they are many and small in size. The informed users have no (strict) incentive to communicate their information to the uninformed users, because the payoff of each user is affected only by his own action. ${ }^{16}$ The uninformed users cannot infer the signal realization from the actions of the informed users before

\footnotetext{
${ }^{14}$ Alternatively, $x$ can be interpreted as the probability that user $i$ observes the signal.

${ }^{15} \mathrm{An}$ additional motivation to focus solely on the information gathered by managers is that prior literature has analyzed the effects of selective disclosure on the incentives of the users to acquire information on their own (Jorgensen, Li and Melumad, 2011).

${ }^{16}$ I assume the users cannot make side payments and collude on the information.
} 


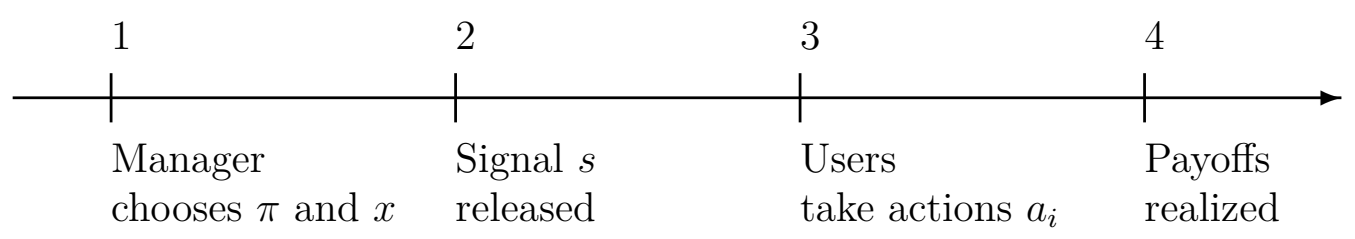

Figure 1: Timeline of events

choosing their actions, because all actions are taken simultaneously.

Figure 1 shows the timeline of the events. At date 1, the manager chooses the design of information system $\pi$ and the fraction $x$ of informed users (under UD). At date 2, the information system reveals signal $s$ to the fraction $x$ of informed users. At date 3, the users take actions, and, at date 4, the payoffs are realized.

\section{Access to Information}

I solve the model by backward induction. To avoid confusion I use a subscript $t=1$ for the expectation operator to denote the expectation at date 1 over the random variables $\omega$ and $s$ and a subscript $t=3$ to denote the posterior expectation at date 3 of $\omega$ after observing the realization of the signal $s$. At date 3 , after observing the choice of information system and the signal realization, the informed users form Bayesian rational beliefs regarding the state of nature and, given the quadratic loss nature of their payoff functions, take actions that equal the posterior expectation:

$$
\hat{a}(s, \pi) \equiv \arg \max _{a_{i}} E_{t=3}\left[u\left(a_{i}, \omega\right) \mid s, \pi\right]=E_{t=3}[\omega \mid s, \pi]
$$


To avoid clutter I denote $\mu_{s, \pi} \equiv E_{t=3}[\omega \mid s, \pi]$. Lacking information, the uninformed users take actions that equal the prior expectation:

$$
\hat{a}(\emptyset) \equiv \arg \max _{a_{i}} E_{t=3}\left[u\left(a_{i}, \omega\right) \mid \emptyset\right]=E_{t=3}[\omega]=\mu_{0} .
$$

As a result, the aggregate action of the users at date 3 is a weighted average of the posterior (for the informed users) and the prior (for the uninformed users):

$$
\hat{A}(x, s, \pi) \equiv \int_{0}^{x} \hat{a}(s, \pi) d i+\int_{x}^{1} \hat{a}(\emptyset) d i=x \mu_{s, \pi}+(1-x) \mu_{0} .
$$

Then, the expected payoff of the manager at date 1 is given by

$$
V(x, \pi) \equiv E_{t=1}[v(\hat{A}(x, s, \pi), \omega)] .
$$

Firm managers choose the precision of the information system that they implement, but they cannot always choose the fraction of users who observe the information. For example, even when regulators gravitate to rules that ensure equal access to information, not all users may observe the available information for various exogenous reasons. It is therefore useful to consider, as a benchmark, the case of exogenous fraction of informed users. At date 1, the manager chooses the information system to maximize her expected utility:

\section{Program $\mathcal{P}^{e x o g}$}

$$
\begin{array}{ll} 
& \max _{\pi} V(x, \pi) \\
\text { s.t. } & \pi_{0}=\int B \pi(d B) .
\end{array}
$$


By implementing an information system the manager "persuades" the users, i.e., she convinces the users to take actions that are closer to her preferred actions and differ from the actions the users would have taken without the information. Kamenica and Gentzkow (2011) find that a sender-in my model, the managerbenefits from persuading a single user if her expected payoff is convex in the user's beliefs regarding the state of nature of the firm. Lemma 1 and Corollary 1 translate this result to my setting and extend it to multiple users, a fraction $x$ of whom are informed for exogenous reasons.

Lemma 1 Suppose $x \in[0,1]$ is exogenously given. If $k>\frac{x}{2}\left(k \leq \frac{x}{2}\right)$ the manager's ex-ante payoff increases (decreases) in the variance of the users' posterior expectation $\mu_{s, \pi}$.

Corollary 1 The manager implements a perfectly revealing information system if and only if $k>\frac{x}{2}$. Otherwise, she does not implement an information system.

The formal proofs are omitted as they follow from Kamenica and Gentzkow (2011) and the discussion below. Note that at date 3, after observing the signal, the manager and the users share the same beliefs. Then, by the Law of Iterated Expectations, the manager's expected payoff at date 1 can be conveniently presented 
as:

$$
\begin{aligned}
& V(x, \pi)=\underbrace{E_{t=1}[\underbrace{E_{t=3}[v(\hat{A}(x, s, \pi), \omega)]}_{\text {posterior expectation at date } 3]}}_{\text {expectation over signal realizations at date } 1} \\
& =E_{t=1}\left[E_{t=3}\left[-\left(x \mu_{s, \pi}+(1-x) \mu_{0}-k \omega-(1-k) \bar{\omega}\right)^{2}\right]\right] \\
& =E_{t=1}\left[E_{t=3}\left[-\left(x \mu_{s, \pi}-k \omega\right)^{2}\right]\right] \\
& -\left[(x-2 k+1) \mu_{0}-(1-k) \bar{\omega}\right]\left[(1-x) \mu_{0}-(1-k) \bar{\omega}\right] \\
& =\underbrace{E_{t=1}\left[2 x\left(k-\frac{x}{2}\right) \mu_{s, \pi}^{2}\right]}_{\text {term } 1}-\underbrace{k^{2}\left(\mu_{0}^{2}+\operatorname{Var}(\omega)\right)}_{\text {term } 2=\text { const }} \\
& -\underbrace{\left[(x-2 k+1) \mu_{0}-(1-k) \bar{\omega}\right]\left[(1-x) \mu_{0}-(1-k) \bar{\omega}\right]}_{\text {term } 3=\text { const }} .
\end{aligned}
$$

The second and third terms in (6) are constant across signal realizations, s. However, term 1 depends on the posterior expectation that a signal induces, and the manager can control its magnitude by choosing the information system. Noting that term 1 is an expectation of a quadratic function of the random variable $\mu_{s, \pi}$ whose expectation equals the prior $\mu_{0}$ and, using Jensen's inequality,

$$
E_{t=1}\left[2 x\left(k-\frac{x}{2}\right) \mu_{s, \pi}^{2}\right]\left\{\begin{array}{l}
>2 x\left(k-\frac{x}{2}\right) \mu_{0}^{2} \geq 0 \quad \text { if } k>\frac{x}{2}, \\
<2 x\left(k-\frac{x}{2}\right) \mu_{0}^{2} \leq 0 \text { if } k<\frac{x}{2} .
\end{array}\right.
$$

If the preferences of the manager are sufficiently aligned with those of the users $\left(k>\frac{x}{2}\right)$, then the manager's payoff is convex in $\mu_{s, \pi}$, and the manager is better off inducing a nontrivial posterior expectation than leaving the users with their prior expectation. The opposite logic holds when the preferences of the manager 
are sufficiently misaligned with those of the users $\left(k<\frac{x}{2}\right)$. To gain further insight note that using (6),

$$
\begin{aligned}
V(x, \pi) & =E_{t=1}\left[2 x\left(k-\frac{x}{2}\right) \mu_{s, \pi}^{2}\right]+\text { const } \\
& =2 x\left(k-\frac{x}{2}\right)\left(\operatorname{Var}\left(\mu_{s, \pi}\right)+\mu_{0}^{2}\right)+\text { const }
\end{aligned}
$$

where $\operatorname{Var}\left(\mu_{s, \pi}\right)$ is the variance of the posterior expectation. When the preferences of the players are aligned $\left(k>\frac{x}{2}\right)$, the manager wants to induce as much variance as possible, which she achieves by implementing the most informative system. Under the specific assumptions of the model this system is fully revealing. ${ }^{17}$ The opposite is true when the preferences of the players are misaligned $\left(k \leq \frac{x}{2}\right)$. The manager then minimizes the variance by not implementing information system. ${ }^{18}$ In the knife-edge case, when $k=\frac{x}{2}$, the manager's expected payoff is the same regardless of the variance in posterior expectation that she induces. For the remainder of the paper, I assume that, whenever the manager is indifferent, she does not implement an information system.

As a next step, I consider the manager's ability to control the aggregate information flow by limiting access to information of a subset of users. To do so, I relax the assumption that $x$ is exogenously given and let the manager choose not only the information system precision but also the fraction of informed users (regime UD). At date 1, the full-fledged optimization problem of the manager is

\footnotetext{
${ }^{17}$ To see why note that, by the Law of Total Variance, $\operatorname{Var}\left(\mu_{s, \pi}\right)=\operatorname{Var}(\omega)-E_{t=3}[\operatorname{Var}(\omega \mid s)]$. Hence, $\operatorname{Var}\left(\mu_{s, \pi}\right) \in[0, \operatorname{Var}(\omega)]$ and achieves maximum when the information system is fully revealing (if $s=\omega$, then $E_{t=3}[\operatorname{Var}(\omega \mid s)]=0$ ).

${ }^{18}$ As then $E_{t=3}[\operatorname{Var}(\omega \mid s)]=\operatorname{Var}(\omega)$ and $\operatorname{Var}\left(\mu_{s, \pi}\right)=0$.
} 


\section{Program $\mathcal{P}^{U D}$}

$$
\begin{array}{ll} 
& \max _{\pi, x \in[0,1]} V(x, \pi) \\
\text { s.t. } & \pi_{0}=\int B \pi(d B) .
\end{array}
$$

Let $(\hat{x}, \hat{\pi})$ denote the solution to this program. Simplifying, the expected utility of the manager can be presented as:

$$
\begin{aligned}
V(x, \pi)= & E_{t=1}\left[-\left(x \mu_{s, \pi}+(1-x) \mu_{0}-k \omega-(1-k) \bar{\omega}\right)^{2}\right] \\
= & -\operatorname{Var}\left(x \mu_{s, \pi}+(1-x) \mu_{0}-k \omega-(1-k) \bar{\omega}\right) \\
& -\left(E_{t=1}\left[x \mu_{s, \pi}+(1-x) \mu_{0}-k \omega-(1-k) \bar{\omega}\right]\right)^{2} \\
= & -\operatorname{Var}\left(x \mu_{s, \pi}-k \omega\right)-(1-k)^{2}\left(\mu_{0}-\bar{\omega}\right)^{2} .
\end{aligned}
$$

Given that $-(1-k)^{2}\left(\mu_{0}-\bar{\omega}\right)^{2}$ is constant in $x$ and $\pi$, maximizing $V(x, \pi)$ boils down to minimizing $\operatorname{Var}\left(x \mu_{s, \pi}-k \omega\right)=E_{t=1}\left[\left(x \mu_{s, \pi}-k \omega\right)^{2}\right]-(x-k)^{2} \mu_{0}^{2}$.

Proposition 1 Under UD, for any $k$, it is optimal for the manager to implement an information system that perfectly reveals the state of nature to a fraction $\hat{x}=k$ of users.

The driving force behind this result is that $V(x) \equiv V(x, \hat{\pi}(x))$ is single peaked at $x=k$. Proposition 1 implies that the manager finds it optimal to restrict access to information. ${ }^{19}$ To better understand the intuition, recall that the aggregate action

\footnotetext{
${ }^{19}$ Allowing for different sensitivity of a representative user's bliss point to the state of nature does not change this result qualitatively as long as the manager prefers smoother aggregate action across states. Under this scenario the optimal fraction of informed users equals the ratio of the sensitivities of the manager's and a representative user's bliss points. Proof available upon request.
} 
of the users at date 3 is as stated in equation (5):

$$
\hat{A}(x, s, \pi)=x \mu_{s, \pi}+(1-x) \mu_{0}
$$

while the manager would want it to be as close as possible to her bliss point as stated in equation (2):

$$
A^{*}(\omega)=k \omega+(1-k) \bar{\omega}
$$

for any realization of $\omega$. The objective of the manager is to minimize the difference between $\hat{A}(x, s, \pi)$ and $A^{*}(\omega)$. By acquiring information about $\omega$, the manager cannot control the difference between $(1-x) \mu_{0}$ and $(1-k) \bar{\omega}$. But she can minimize the difference between $x \mu_{s, \pi}$ and $k \omega$. Implementing a partially informative system and providing the information to more users will change the term $x \mu_{s, \pi}$ into an ex ante (at date 1) unknown direction. By implementing a perfectly informative system and revealing the signal to exactly a fraction $k$ of users, the manager controls $x \mu_{s, \pi}$ and makes sure that ex-post it is exactly $k \omega$.

Put differently, if the state of nature were observable, the manager would want the users to react to the realization of $\omega$ with a response coefficient of $k$ (as, by $\left.(2), \frac{d A^{*}(\omega)}{d \omega}=k\right)$. In the model, the users only get to see the signal, $s$, and not the state, $\omega$, directly. In order to regulate the sensitivity with which the users react to the signal, which, by $(5)$, is $\frac{d \hat{A}(x, s, \pi)}{d s}=x \frac{d \hat{a}(s, \pi)}{d s}+\frac{d \hat{a}(\emptyset)}{d s}$, the manager has two instruments at her disposal: $\pi$, which determines the response coefficient $\frac{d \hat{a}(s, \pi)}{d s}$ for an informed user, and $x$, which determines the fraction of informed users. ${ }^{20}$ One way to implement an expected response coefficient of $k$ is to set $x=1$ and

\footnotetext{
${ }^{20}$ The uninformed users, by default, have response coefficient $\frac{d \hat{a}(\emptyset)}{d s}=0$.
} 
$\pi$ (calculated as a plug) that ensures $\frac{d \hat{a}(s, \pi)}{d s}=k$. This, however, introduces noise into the signal, which is costly to the users and to the manager. Alternatively, the manager can set $x=k$ and implement a perfectly informative information system. This would yield the same response coefficient in expectation but avoids the noise in the signal, which makes this the optimal solution.

Note that, by Proposition 1, when the manager is allowed to choose the group of informed users, she always finds it optimal to implement an information system, regardless of the preference misalignment between the players. This result is in stark contrast to the result for exogenously given fraction of informed users in Corollary 1. Technically speaking, this difference arises because the expected payoff of the manager at the optimal fraction $\hat{x}=k$ is always convex in the users' beliefs. Therefore, as described in the discussion following Lemma 1, the manager benefits from introducing variance in the posterior beliefs and maximizes this variance by sending a signal that perfectly reveals the state of nature.

The intuition behind this result is that the choice of $x$ is a tool that regulates the flow of information and enables the manager to persuade the users to take an aggregate action that is as close as possible to her most preferred action. In other words, the ability to limit access to information mitigates the reluctance to implement an information system due to preference misalignment between the players. As a result, the manager always finds it optimal to implement a system that releases a perfectly informative signal to the optimally chosen fraction of users.

Regulators often gravitate to rules that ensure equal access to information for all agents in an economy. To consider the implications of such rules, I compare two alternative regimes: mandated dissemination (MD), under which the manager 
is required to disclose information to all users, i.e., $x=1$ is exogenously set, ${ }^{21}$ and unregulated dissemination (UD), under which the manager can optimally choose the fraction of informed users in her own best interest.

\section{Proposition 2}

(i) If $k \leq \frac{1}{2}$, UD Pareto dominates $M D$.

(ii) If $k>\frac{1}{2}$, the manager is better off under UD, but all users are at least weakly better off under $M D$.

The formal proof is omitted as it follows from the discussion below. Under UD, the manager can choose any fraction $x \in[0,1]$, but she finds it optimal to limit access to information to some users (i.e., sets $\hat{x}=k<1$ ). Hence, by revealed preference, she always prefers UD over MD. The users' preference is less obvious. At the heart of the comparison lie the observations that, whenever the manager chooses to implement an information system, she implements a fully informative one and that, given their quadratic loss payoff, the users benefit from fully revealing information.

By Corollary 1 and Proposition 1 , if $k \leq \frac{1}{2}$, then the manager implements an information system only under UD. The informed users are better off under UD (because it is the only regime under which the manager implements an information system). The uninformed users are indifferent (because they do not observe information under either regime). As a result, UD Pareto dominates MD. However, if $k>\frac{1}{2}$, the manager implements a perfectly revealing information system under both regimes. The informed users are indifferent (because they perfectly observe

\footnotetext{
${ }^{21}$ In many cases, even if the regulator sets $x=1$, the actual fraction of informed users is strictly lower as some users will not observe the information (for example, unsophisticated investors). Considering this possibility does not change qualitatively the results.
} 
$\omega$ under both regimes), while the users who are uninformed under UD are better off under MD (because it allows them to observe information).

Paradoxically, Proposition 2 shows that, when the players preferences over the actions are misaligned, their preferences over regimes are aligned, and vice versa. If regulators believe that firm managers and users have very different objectives, then by part (i), they should gravitate to UD, because it Pareto dominates MD. If

$k>\frac{1}{2}$, Pareto ranking of the regimes is not possible, but MD ensures all users are at least weakly better off. Hence, if regulators care exclusively about the welfare of the users and believe the objectives of the managers and the users are sufficiently aligned, they should gravitate to MD.

The result of Proposition 2 may be surprising at first, because conventional wisdom would say that, if the preferences of the players are misaligned, then there is need for the regulator to intervene as players will not arrive at a socially efficient result on their own, and vice versa. However, my model predicts exactly the opposite-the regulator's intervention when the players objectives are misaligned may suppress socially beneficial information acquisition.

A natural question that arises is what is the socially optimal fraction of informed users. I examine this question in subsection 4.4 after I extend the model to account for costly information system implementation.

\section{Costly Persuasion}

In Section 3, the information system under MD has a bang-bang character. This result does not seem particularly descriptive. Therefore, in this section, I extend the results by introducing a cost borne by the manager associated with the imple- 
mentation of the information system. ${ }^{22}$ I show that when implementation is costly the manager sacrifices some amount of precision, and, under standard regularity conditions, her choice is an interior solution. This allows (i) for analyzing how the ability of the manager to limit access to information affects the information system and (ii) for providing new predictions.

To keep the analysis tractable, I impose normality on the distributions of $\omega$ and $s$ for the remainder of the paper:

$$
\begin{aligned}
& \omega=\mu_{0}+\varepsilon, \quad \varepsilon \sim \mathcal{N}\left(0, \frac{1}{\alpha}\right), \quad \alpha \geq 0 \\
& s=\omega+\delta, \quad \delta \sim \mathcal{N}\left(0, \frac{1}{\beta}\right), \quad \beta \geq 0 .
\end{aligned}
$$

The error terms $\varepsilon$ and $\delta$ are independent:

$$
\operatorname{Cov}(\varepsilon, \delta)=0
$$

Upon observing the signal realization, the players form a posterior belief regarding the state of nature:

$$
\omega \mid s \sim \mathcal{N}\left(\frac{\alpha \mu_{0}+\beta s}{\alpha+\beta}, \frac{1}{\alpha+\beta}\right) .
$$

Under these assumptions, the manager's choice of a Bayes-plausible distribution $\pi$ over posterior beliefs simplifies to a choice of $\beta$, which represents the precision of the signal. A choice of $\beta \rightarrow 0$ means the signal does not convey any information,

\footnotetext{
${ }^{22}$ Information system implementation costs are frequently observed in practice. For example, providing a forecast requires hiring an economist, inventory management requires purchasing software, evaluating an asset's fair value requires paying for an appraisal, etc.
} 
while a choice of $\beta \rightarrow \infty$ indicates a signal that fully reveals the realization of $\omega .^{23}$ The manager's choice of $\beta$ is observed by the users. ${ }^{24}$ The prior expectation of the state of nature $\mu_{0}$ and the variance $\frac{1}{\alpha}$ are common knowledge.

Under the normality assumption at date 3 the informed users take actions $\hat{a}(s, \beta)=\mu_{s, \beta}=\frac{\alpha \mu_{0}+\beta s}{\alpha+\beta}$. The expected utility of the manager at date 1 can be presented as:

$$
\begin{aligned}
V(x, \beta) & =E_{t=1}\left[-\left(x \mu_{s, \beta}+(1-x) \mu_{0}-k \omega-(1-k) \bar{\omega}\right)^{2}\right] \\
& =-E_{t=1}\left[\left(x(\varepsilon+\delta) \frac{\beta}{\alpha+\beta}-k \varepsilon+(1-k)\left(\mu_{0}-\bar{\omega}\right)\right)^{2}\right] \\
& =-E_{t=1}\left[\left(x(\varepsilon+\delta) \frac{\beta}{\alpha+\beta}-k \varepsilon\right)^{2}\right]-(1-k)^{2}\left(\mu_{0}-\bar{\omega}\right)^{2}
\end{aligned}
$$

I assume that the manager bears an implementation cost $C(c, \beta)$ with

$$
C_{c}(.) \geq 0, \quad C_{\beta}(.) \geq 0, \quad C_{c c}(.) \geq 0, \quad C_{\beta \beta}(.) \geq 0, \quad C_{c \beta}(.)>0 .
$$

The parameter $c>0$ represents the cost of information system technology in the economy and might be a function of the level of competition in that market. I assume $c$ is exogenously given and refer to it as the "information cost" or just "cost." To ensure interior solutions I assume that

$$
\lim _{\beta \rightarrow \infty} C_{\beta}(c, \beta) \rightarrow \infty \text { and } \lim _{\beta \rightarrow 0} C_{\beta}(c, \beta)=0
$$

\footnotetext{
${ }^{23}$ I do not restrict the precision of the information system, i.e., I assume the manager can implement a fully revealing information system. In many settings there could be an upper bound $\bar{\beta} \in(0, \infty)$ on the precision.

${ }^{24}$ For example, the hire of an economist, the choice of inventory management software, etc., are often publicly observable, and their precision is commonly known.
} 
for any cost. ${ }^{25}$ The total implementation cost is zero whenever information is cost-free or information system is completely imprecise, i.e.,

$$
\lim _{c \rightarrow 0} C(c, \beta)=\lim _{\beta \rightarrow 0} C(c, \beta)=0 .
$$

Lastly, it seems realistic to think that, once the information system is implemented, the associated cost is independent of the fraction of users observing the signal. Assuming otherwise, i.e., that the implementation cost is increasing in the number of the users to which the signal is conveyed (which is the reasonable alternative assumption), would only mechanically facilitate finding that it is optimal for the manager to restrict access to information without qualitatively changing the results.

\subsection{The Optimal Precision}

Similar to the analysis with cost-free implementation, I start by considering the manager's problem when $x \in[0,1]$ is exogenously given. Let

$$
\Delta x \equiv|x-k|
$$

denote the distance between the fraction of informed users and the preference misalignment. At date 1, the manager chooses

$$
\beta^{c}(x) \in \arg \max _{\beta} V(x, \beta)-C(c, \beta),
$$

\footnotetext{
${ }^{25}$ This is the case for many commonly used cost functions, including the quadratic cost function $\frac{c \beta^{2}}{2}$ as a special case. All results hold qualitatively with few minor adjustments for cost functions with $\lim _{\beta \rightarrow 0} C_{\beta}(c, \beta)>0$ (linear cost function $c \beta$ as a special case), i.e., for which the marginal cost from implementing a system with even very small precision is positive. Whenever applicable, I will outline in a footnote the minor adjustments needed under the assumption that $\lim _{\beta \rightarrow 0} C_{\beta}(c, \beta)>0$. Full analysis available upon request.
} 
where the superscript "c" denotes costly information system implementation. ${ }^{26}$

Lemma 2 Suppose $x \in[0,1]$ is exogenously given and the manager bears an implementation cost $C(c, \beta)$. The manager then implements an information system with precision $\beta^{c}(x) \in(0, \infty)$ if and only if $k>\frac{x}{2} . \beta^{c}(x)$ is decreasing in $c$ and in $\Delta x$ and increasing in $\frac{1}{\alpha}$ and in $k$.

Similar to the results in Lemma 1 and Corollary 1 for cost-free implementation, the manager implements an information system if and only if her preferences are sufficiently aligned with those of the users. ${ }^{27}$ The cutoff for implementation $\left(k>\frac{x}{2}\right)$ does not depend on the cost. If $c \rightarrow 0$, then $\beta^{c}(x) \rightarrow \infty$ and therefore the results from the preceding section are recouped as a special case. However, as long as $c>0$, the manager finds it optimal to sacrifice some precision. The higher the cost, the less precise the information system that the manager implements. In the limit, as $c \rightarrow \infty, \beta^{c}(x) \rightarrow 0$, i.e., if the implementation is extremely costly, the signal will be uninformative.

The closer the exogenous fraction $x$ to the preference alignment $k$, the higher the precision of the information system that the manager implements. This is graphically shown in Figure 2. The intuition for this result is that, when $x=k$, the players' preference misalignment is minimized, and this provides stronger incentives for the manager to gather information. The more aligned the manager's preferences with those of the users, the more precise the information system she implements, so that the users' actions will be more in line with the realization of $\omega$. This intuition

\footnotetext{
${ }^{26}$ The optimal precision $\beta^{c}(x)$ depends, in addition to $x$, on other exogenous parameters $c, \alpha$ and $k$. I depress them to avoid clutter.

${ }^{27}$ If $\lim _{\beta \rightarrow 0} C_{\beta}(c, \beta)>0$, i.e., if the implementation of an information system with even very small precision is costly, then the manager will implement an information system only if $c$ is below a certain threshold. It can be shown that the threshold is decreasing in $\Delta x$. Analysis available upon request.
} 


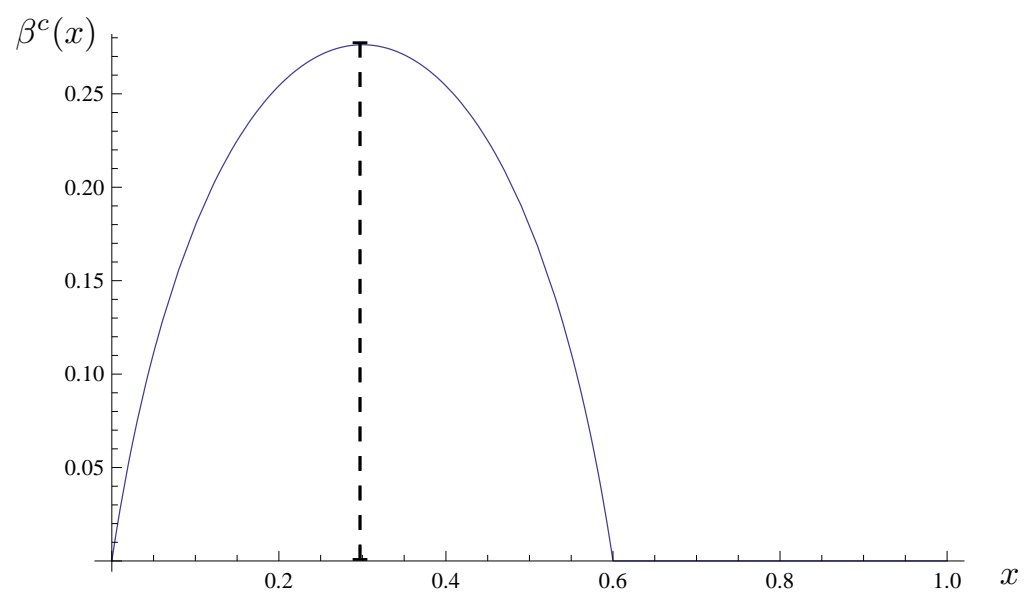

Figure 2: Optimal precision as a function of $x$ Numerical example with $C(c, \beta)=\frac{c \beta^{2}}{2}, \alpha=1, c=0.2, k=0.3$

is shown graphically in Figure 3. As shown in Lemma 2, the signal precision is

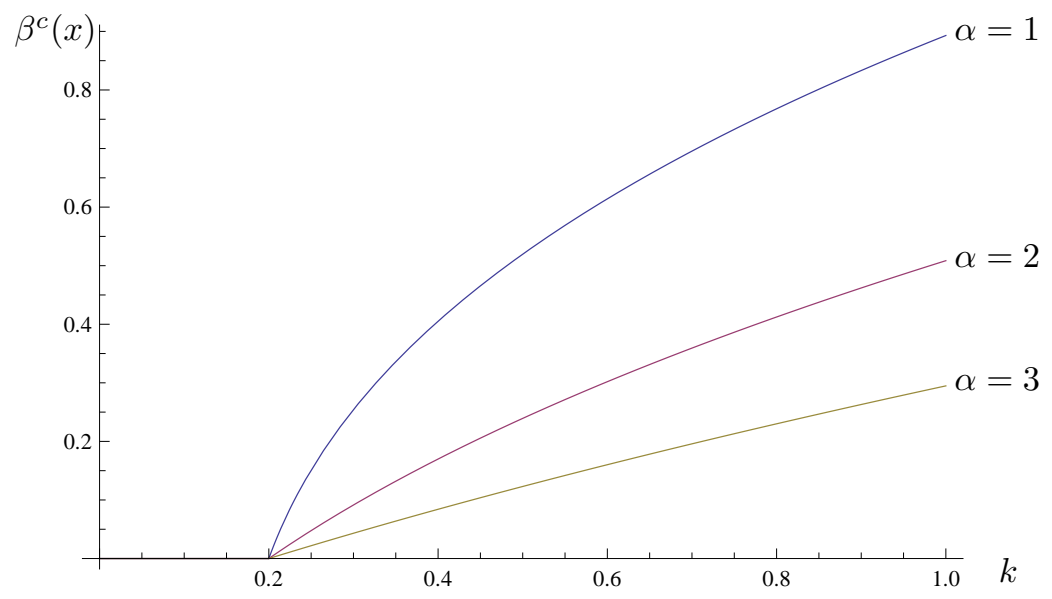

Figure 3: Optimal precision as a function of $k$ Numerical example with $C(c, \beta)=\frac{c \beta^{2}}{2}, c=0.2, x=0.4$

increasing in $\frac{1}{\alpha}$, the prior variance of $\omega$. Further, the optimal precision does not depend on $\bar{\omega}$. This confirms the previously noted observation that the manager cannot persuade the users to take an action close to $\bar{\omega}$. 
As a next step, I consider the ability of the manager to limit access to information to a subset of users by optimally choosing $x$. At date 1 , the manager's full-fledged optimization problem is:

Program $\mathcal{P}^{c, U D}$

$$
\max _{\beta \geq 0, x \in[0,1]} V(x, \beta)-C(c, \beta) .
$$

Let $\left(x_{U D}^{c}, \beta_{U D}^{c}\right)$ denote the solution to this program. The result below extends Proposition 1 to the costly implementation setting.

Proposition 3 Suppose the manager bears an implementation cost $C(c, \beta)$. Under $U D$, for any $k$, it is optimal for the manager to implement an information system with precision $\beta_{U D}^{c} \equiv \beta^{c}(x=k)$ and to disseminate the signal to a fraction $x_{U D}^{c}=k$ of informed users.

The key finding here is that the optimal subset of informed users is unaffected by the cost. The choice of $x$ is a device that induces the right aggregate signal-response coefficient of $k$, and that logic is unaffected by any implementation costs. However, when implementation is costly, the manager implements an information system that provides a noisy signal. The comparative statics of the optimal precision with respect to $\frac{1}{\alpha}, c, k$ and $\bar{\omega}$ are similar in nature to those of $\beta^{c}(x)$.

\subsection{Regime Preferences}

Straightforward application of Lemma 2 reveals that under MD the manager implements an information system with precision

$$
\beta_{M D}^{c} \equiv \beta^{c}(x=1)>0
$$


if $k>\frac{1}{2}$ and zero otherwise. By Lemma 2, the information system implemented under UD is more precise than the one implemented under MD:

$$
\Delta \beta \equiv \beta_{U D}^{c}-\beta_{M D}^{c}>0
$$

Similar to the cost-free setting, the manager always prefers (by revealed preference) UD, and the uninformed users are always weakly better off under MD. ${ }^{28}$ However, the comparison with regards to the informed users is more complicated and requires taking into account that the equilibrium precisions under both regimes are different as shown in (10) and that the users want as precise information as possible. The analysis shows that the informed users are always strictly better off under UD, because it ensures they observe a more precise signal. The result below extends Proposition 2 to the costly implementation setting.

Proposition 4 Suppose the manager bears an implementation cost $C(c, \beta)$.

(i) If $k \leq \frac{1}{2}$, UD Pareto dominates $M D$.

(ii) If $k>\frac{1}{2}$, the uninformed users are strictly better off under $M D$, while the informed users and the manager are strictly better off under UD.

As before, if $k \leq \frac{1}{2}$, UD Pareto dominates MD. Hence, if regulators believe the preferences of firm managers and users in an economy are sufficiently misaligned, they should not enforce equal access to information of all potential users. If $k>\frac{1}{2}$, there is an additional dimension to the disagreement issue discussed in the cost-free setting, because now even the different types of users, endogenously divided into informed and uninformed ones, prefer different regimes.

\footnotetext{
${ }^{28}$ If $k>\frac{1}{2}$, it is the only regime that enables them to observe a signal, while if $k \leq \frac{1}{2}$, then they are indifferent because they do not observe a signal under either regime.
} 


\subsection{Welfare Analysis with Sufficiently Aligned Preferences}

Proposition 4 shows that, when the preferences of the players are sufficiently aligned, neither of the regimes ensures all users are at least weakly better off simultaneously. In this subsection, I conduct welfare analysis to evaluate under which regime the users are better off at an aggregate level when $k>\frac{1}{2}$. Let

$$
\phi^{c}(x) \equiv E_{t=1}\left[u\left(\hat{a}\left(s \mid \beta^{c}(x)\right), \omega\right)\right]-E_{t=1}[u(\hat{a}(\emptyset), \omega)]
$$

denote a representative user's expected gain of becoming informed (hereafter, "information gain"). Then,

$$
\begin{aligned}
W^{c}(x) & =\int_{0}^{x} E_{t=1}\left[u\left(\hat{a}\left(s \mid \beta^{c}(x)\right), \omega\right)\right] d i+\int_{x}^{1} E_{t=1}[u(\hat{a}(\emptyset), \omega)] d i \\
& =\int_{0}^{1} E_{t=1}[u(\hat{a}(\emptyset), \omega)] d i+\int_{0}^{x} \phi^{c}(x) d i \\
& =\underbrace{E_{t=1}[u(\hat{a}(\emptyset), \omega)]}_{\text {base welfare }}+\underbrace{x \phi^{c}(x)}_{\text {aggregate gain }}
\end{aligned}
$$

is the aggregate users' welfare. ${ }^{29}$ The base welfare represents the aggregate payoff of all users when the manager does not implement an information system. The aggregate gain is the information gain of the informed users, on an aggregate level. While the base welfare is independent of $x$, the comparative statics of the aggregate gain with respect to $x$ is a priori unclear. As seen from (11), there are two effects: (i) a direct effect-as $x$ increases, more users benefit from information, and (ii) an indirect effect-the fraction $x$ affects the information gain indirectly through the optimal precision.

\footnotetext{
${ }^{29}$ The gain $\phi^{c}(x)$ and the aggregate welfare $W^{c}(x)$ depend, in addition to $x$, on other exogenous parameters: $c, \alpha$ and $k$. I depress them to avoid clutter.
} 
Corollary $2 \phi^{c}(x)$ is decreasing in $\Delta x \equiv|x-k|$.

The intuition behind this result is that the information gain is increasing in the precision, which is single-peaked at $x=k$ by Lemma 2 . As a result, the informed users are better off when the manager discloses information only to a fraction $k$ of users. Equation (11) and Corollary 2 imply that broader dissemination of information is not always better for the users on an aggregate level. To compare the users' welfare under both regimes, let

$$
\begin{gathered}
\phi_{M D}^{c} \equiv \phi^{c}(x=1) \\
\phi_{U D}^{c} \equiv \phi^{c}(x=k)
\end{gathered}
$$

denote the information gain of a representative informed user and

$$
\begin{aligned}
& W_{M D}^{c} \equiv W^{c}(x=1) \\
& W_{U D}^{c} \equiv W^{c}(x=k)
\end{aligned}
$$

the aggregate welfare of the users under MD and UD, respectively. Comparing UD and $\mathrm{MD}$ on an aggregate level requires signing the welfare differential

$$
\Delta W \equiv W_{U D}^{c}-W_{M D}^{c}=\underbrace{k \phi_{U D}^{c}}_{\text {aggregate gain UD }}-\underbrace{\phi_{M D}^{c}}_{\text {aggregate gain MD }}
$$

The comparison of the aggregate gains is affected by two countervailing effects: (i) an omission effect-under UD, the proportion of informed users is lower than the proportion under MD $\left(x_{U D}^{c}=k<1\right)$-and (ii) a precision effect-by Lemma 2, the signal precision under UD is higher than the one under MD. As a result, by 
Corollary 2, the information gain under UD is larger than the one under MD:

$$
\Delta \phi \equiv \phi_{U D}^{c}-\phi_{M D}^{c}>0
$$

The next result presents sufficient conditions for the aggregate welfare under UD to exceed the one under MD.

Proposition 5 Suppose $k>\frac{1}{2}$ and the manager bears an implementation cost $C(c, \beta)=\frac{c \beta^{2}}{2}$. Then there exist $\tilde{k} \in\left(\frac{1}{2}, 1\right)$, such that $W_{U D}^{c} \geq W_{M D}^{c}$ if $k \leq \tilde{k}$, and $c$ is sufficiently high.

This result is graphically shown in Figure 4. As $c \rightarrow 0$, the manager sets the same (infinite) precision under UD and MD. Hence the gain from information of a representative informed user under both regimes is the same, and $\Delta W \propto k-1<0$ so that the users prefer $\mathrm{MD}$, in aggregate. As $c$ increases, the optimal precision and, as a result, the respective aggregate gain decrease. As $c \rightarrow \infty$, the aggregate gain under both regimes reaches zero. However, as I show in the proof, the gain under MD does so faster than the one under UD if the preference alignment is sufficiently bounded away from one. ${ }^{30}$ As a result, for some sufficiently high cost, the aggregate gain under MD is larger than the aggregate gain under UD.

Another way to look at the efficiency effects of the manager's ability to limit access to information is by considering the accuracy of the aggregate users' action, i.e., the distance between the aggregate action and the state of nature. In the leading example, this reflects the accuracy of the consensus forecast, observed by

\footnotetext{
${ }^{30}$ When the preference alignment is sufficiently close to one, the aggregate gains under both regimes are of similar magnitude and decrease in $c$ at the same rate.
} 


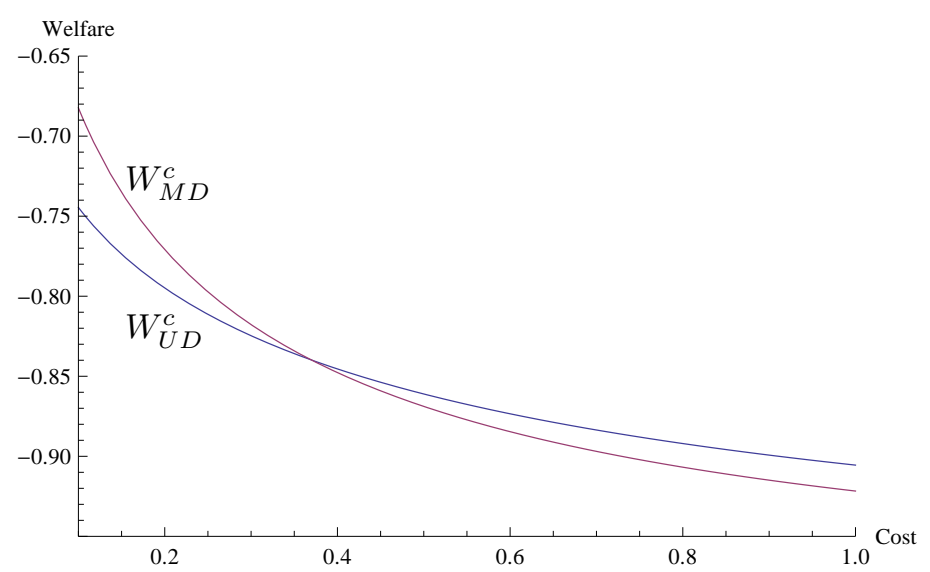

Figure 4: Users' welfare comparison Numerical example with $C(c, \beta)=\frac{c \beta^{2}}{2}, \alpha=1, k=0.55$

investors. ${ }^{31}$ It can be shown that the conditions ensuring users are better off, on an aggregate level, under UD are sufficient for the aggregate action to be more accurate. $^{32}$

\subsection{Regulated Information Dissemination}

I conclude the analysis by discussing the socially optimal fraction of informed users. To do so, I assume that a benevolent regulator ("he") chooses the subset of users who get to observe the signal prior to the manager's choice of information system and call this regime regulated dissemination ("RD"). The regulator chooses $x$ to maximize the aggregate expected payoffs of all players, subject to the constraint

\footnotetext{
${ }^{31}$ Earnings forecasts are a main source of earnings information of many investors. Brown and Rozeff (1978); Fried and Givoly (1982); Givoly and Lakonishok (1984); Conroy and Harris (1987); Brown, Hagerman, Griffin and Zmijewski (1987); O'Brien (1988); Kross, Ro and Schroeder (1990) show that forecasts are a good proxy for investors' earnings expectations.
}

${ }^{32}$ The proof is available upon request. 
that the information system precision is chosen by the manager in her own interest:

$$
\begin{array}{ll}
\max _{x \in[0,1]} \lambda\left[V\left(x, \beta^{c}(x)\right)-C\left(c, \beta^{c}(x)\right)\right]+W^{c}(x) \\
\text { subject to } \quad \beta^{c}(x) \equiv \arg \max _{\beta} V(x, \beta)-C(c, \beta) .
\end{array}
$$

The parameter $\lambda \geq 0$ represents the weight that the regulator puts on the manager's welfare. It is straightforward that, if the regulator cares only about the manager's welfare (i.e., $\lambda \rightarrow \infty$ ), he will choose the same fraction of informed users that the manager would have set in her own interest $\left(x_{R D}^{c}=k\right)$. However, if the regulator sufficiently cares about the users' welfare, the socially beneficial level of $x$ may go beyond $k$ and, under certain conditions, may even reach one.

Lemma 3 Suppose $\lambda \leq 1$ and the manager bears an implementation $\operatorname{cost} C(c, \beta)=$ $\frac{c \beta^{2}}{2}$ with $c \geq 0$. Then the socially beneficial fraction of informed users $x_{R D}^{c} \in[k, 1]$ is decreasing in $c$.

When the players' preferences are sufficiently misaligned, the regulator wants to restrict access to information for some users even when he does not care at all about the manager's welfare (i.e., $\lambda=0$ ), because he wants to ensure the manager has incentives to implement an information system and disseminate socially valuable information. ${ }^{33}$ However, when the players preferences are sufficiently aligned and information acquisition is cost-free, the regulator chooses a corner solution for $x$ and enforces equal access to information. The rationale behind this observation is that when $c \rightarrow 0$ the gain in collective users' welfare is larger than the loss in

\footnotetext{
${ }^{33}$ If $k \leq \frac{1}{2}$, then $2 k \leq 1$. To ensure that the manager implements an information system, the planner needs to make sure that $x<2 k \leq 1$. This observation confirms the result in Proposition 4 (i).
} 
manager's payoff caused by increasing $x$ beyond $k{ }^{34}$ Introduction of costs changes the corner solution character of the socially beneficial fraction of informed users. Put differently, unlike the fraction of informed users set by the manager in her own interest, the fraction $x$ that the regulator enforces is decreasing in the implementation cost. The reason for this finding is that, as $c$ increases, the manager chooses a lower precision. Then, to provide incentives for the manager to increase the precision of the socially valuable information, even the users themselves, at some prior state, would collectively agree to some $x<1$, as long as they are behind the veil of ignorance (Harsanyi, 1955), i.e., before each learns whether he will be included in the group of informed users.

\section{$5 \quad$ Biased Users of Information}

In Section 3 and Section 4 the users of information are unbiased as they prefer actions that are fully aligned with the state of nature of the firm. However, the users can sometimes be cautious (aggressive) and take actions that are lower (higher) than the state of nature of the firm. In the leading example, analysts compensation and reputation may be more severely hurt in the case of an upward forecast error than in the case of a downward one. This may provide incentives to the analysts to be more conservative in their forecasts.

I now consider how the introduction of bias in the users' preferences affects the

\footnotetext{
${ }^{34}$ When $k>\frac{1}{2}$, the implementation constraint is satisfied for any $x$, and with $c=0$ the manager implements a perfectly revealing information system. Hence the derivative of the regulator's objective function with respect to $x$ reads $\frac{2 \lambda(k-x)}{\alpha}+\frac{1}{\alpha}$. If $x<k$ both terms are positive, i.e., all players benefit from increasing $x$. If $x>k$, then the first term is negative and represents the decrease in manager's payoff from increasing the fraction of informed users beyond $k$. The second term represents the gain in aggregate users' welfare from an increase in $x$. If $\lambda \leq 1$ and $k>\frac{1}{2}$, then $2 \lambda(k-x)+1>2 k-1>0$, i.e., the gain in users' welfare is larger than the loss in manager's payoff.
} 
prior results. I return to the assumptions maintained in Section 3: (i) the state of nature of the firm is distributed according to some (commonly known) probability distribution $\pi_{o} \in \Delta(\Omega)$, and (ii) the manager can implement a cost-free information system that creates a (commonly known) distribution over posterior beliefs $\pi \in$ $\Delta(\Delta(\Omega))$. Under the assumption that the users are also biased, the parameter $k$ does not capture anymore the preference alignment between the players. Through out this Section I will refer to $k$ as "the manager's bias". The higher $k$ the less biased the manager.

If all users have identical bias then the results in Section 3 remain qualitatively similar except that now the manager's preference bias, $k$, is scaled by the respective users' preference bias. ${ }^{35}$ The more interesting case is when the users' bias is heterogeneous. Given that in practice users tend to differ in their cautiousness (aggressiveness) this scenario also seems to be the more realistic one. In the leading example, this assumption will reflect the heterogeneity in analysts' compensation or sensitivity to reputation.

To analyze the effect of heterogeneous bias I sort the users by their respective bias and assume that the ex post payoff of user $i$ is given by:

$$
u^{H}\left(a_{i}, \omega\right)=-\left(a_{i}-i \omega-(1-i) \underline{\omega}\right)^{2}
$$

\footnotetext{
${ }^{35}$ To illustrate, suppose that the ex post payoff of a representative user is $u\left(a_{i}, \omega\right)=-\left(a_{i}-l \omega-\right.$ $(1-l) \underline{\omega})^{2}$, where $l \in(0,1)$ and $\underline{\omega} \in \mathbb{R}$ are commonly known parameters. Here, $l$ captures the bias of the users. The ratio $\frac{k}{l}$ represents the preference alignment between the players: $\frac{k}{l} \rightarrow 1$ indicates that the preferences of the players are aligned, while $\frac{k}{l}<1\left(\frac{k}{l}>1\right)$ indicates that the manager is more (less) biased than the users. Under UD, the manager implements a perfectly informative information system and disseminates the signal to a fraction $x=\min \left\{\frac{k}{l}, 1\right\}$ of informed users. Put differently, the manager restricts the access to information only when she is more biased than the users (i.e., when $\frac{k}{l}<1$ ). Otherwise, she publicly reveals the information. Detailed analysis available upon request.
} 
where the superscript " $\mathrm{H}$ " denotes "heterogeneous bias", $\underline{\omega} \in \mathbb{R}$ is a parameter and, as before, $i \sim U[0,1]$. For any realization of $\omega$, the interior solution that maximizes the payoff of user $i$ is:

$$
a_{i}^{*}(\omega) \equiv \arg \max _{a_{i}} u^{H}\left(a_{i}, \omega\right)=i \omega+(1-i) \underline{\omega},
$$

i.e., user $i$ prefers an action that is partially aligned with the state of nature of the firm and partially biased towards a specific action $\underline{\omega}$. The higher $i$, the less biased the user. In the limit, as $i \rightarrow 1$, user $i$ becomes unbiased. At date 3 , the informed users take an action

$$
\hat{a}^{H}(s, \pi) \equiv \arg \max _{a_{i}} E_{t=3}\left[u^{H}\left(a_{i}, \omega\right) \mid s, \pi\right]=i \mu_{s, \pi}+(1-i) \underline{\omega}
$$

and the uninformed users take an action

$$
\hat{a}^{H}(\emptyset) \equiv \arg \max _{a_{i}} E_{t=3}\left[u^{H}\left(a_{i}, \omega\right) \mid \emptyset\right]=i \mu_{0}+(1-i) \underline{\omega} .
$$

Let $[\underline{x}, \bar{x}]$ with $0 \leq \underline{x} \leq \bar{x} \leq 1$ represent the partition of users that become informed. ${ }^{36}$ In the following, I will refer to $\int_{\underline{x}}^{\bar{x}} i d i$ as the "aggregate bias of the informed users." The aggregate action is

$$
\begin{aligned}
A^{H}(\underline{x}, \bar{x}, s, \pi) & =\int_{0}^{\underline{x}} \hat{a}^{H}(\emptyset) d i+\int_{\underline{x}}^{\bar{x}} \hat{a}^{H}(s, \pi) d i+\int_{\bar{x}}^{1} \hat{a}^{H}(\emptyset) d i \\
& =\left(\mu_{s, \pi}-\mu_{0}\right) \int_{\underline{x}}^{\bar{x}} i d i+\left(\mu_{0}+\underline{\omega}\right) \int_{0}^{1} i d i
\end{aligned}
$$

\footnotetext{
${ }^{36}$ In the formal analysis I restrict attention to compact intervals of informed users. However, as I will discuss later, the manager cannot increase her payoff by disseminating information to a non-compact subset of users.
} 
and the manager's expected payoff can be presented as

$$
V^{H}(\underline{x}, \bar{x}, \pi) \equiv E_{t=1}\left[v\left(A^{H}(\underline{x}, \bar{x}, s, \pi), \omega\right)\right]
$$

Then, at date 1 the manager solves:

Program $\mathcal{P}^{H}$

$$
\begin{array}{ll} 
& \max _{\pi, \underline{x}, \bar{x}} V^{H}(\underline{x}, \bar{x}, \pi) \\
\text { s.t. } & \pi_{0}=\int B \pi(d B) \\
& 0 \leq \underline{x} \leq \bar{x} \leq 1 .
\end{array}
$$

The result below extends the results of Proposition 1 to the case when the users of information differ in their bias.

Proposition 6 Under UD, the manager implements a perfectly revealing information system.

(i) If $k<\frac{1}{2}$, the manager restricts access to information. There exist a continuum $[\underline{x}, \underline{x}+f(\underline{x})] \subset[0,1]$ that achieves the same maximum payoff to the manager. The function $f(\underline{x})$ is decreasing in $\underline{x}$ and increasing in $k$.

(ii) If $k \geq \frac{1}{2}$, the manager publicly disseminates the signal.

If the state of nature of the firm were observable, the manager would want the users to react to the realization of $\omega$ with a response coefficient of $k$. However, the users only get to see the signal and, by (13), react to it with $\frac{d A^{H}(\underline{x}, \bar{x}, s, \pi)}{d s}=\int_{\underline{x}}^{\bar{x}} i d i \frac{d \mu_{s, \pi}}{d s}$. To regulate the users' sensitivity the manager implements a perfectly informative 
system (so that $\frac{d \mu_{s, \beta}}{d s}=1$ ) and reveals the signal to a partition $[\underline{x}, \bar{x}]$ of users such that:

$$
\underbrace{k}_{\text {manager's bias }}=\underbrace{\int_{x}^{\bar{x}} i d i}_{\text {informed users' aggregate bias }}
$$

Put differently, the manager chooses the group of informed users so that their aggregate bias is aligned with her own bias. ${ }^{37}$ If the manager is severely biased $\left(k<\frac{1}{2}\right)$, there are different subsets of users with such aggregate bias. ${ }^{38}$ If $k=\frac{1}{2}$, the manager disseminates globally because her preferences are perfectly aligned with the aggregate bias of all existing users. ${ }^{39}$ If the manager is mildly biased $\left(k>\frac{1}{2}\right)$, then she is more biased than the existing users, collectively. Therefore, she wants to reveal the signal to as many users as possible. The best she can do is to disseminate the signal globally. ${ }^{40}$

It is useful to consider the comparative statics of the size of the fraction of informed users, represented by $f(\underline{x})$, for a given $\underline{x}$. When the least biased informed user is only mildly biased (i.e., when $\underline{x}$ is low), the manager disseminates the signal to fewer users. ${ }^{41}$ The intuition behind this result is that less biased users react with higher sensitivity to the signal and the manager needs fewer users "in the loop" to

\footnotetext{
${ }^{37}$ To do so, the manager need not restrict herself only to a compact subset of users. However, any non-compact subset of users that achieves an aggregate sensitivity of $k$ will lead to the same maximum payoff to the manager.

${ }^{38}$ If $k<\frac{1}{2}$, there are multiple $(\underline{x}, \bar{x})$ that satisfy (14).

${ }^{39}$ When $k=\frac{1}{2}$, only $\underline{x}=0$ and $\bar{x}=1$ satisfy (14).

${ }^{40}$ If $k>\frac{1}{2}$, condition (14) can not be satisfied for any $\underline{x} \geq 0$ and $\bar{x} \leq 1$ because, by assumption, $0 \leq \underline{x} \leq \bar{x} \leq 1$, so the right hand side of (14), $\int_{\underline{x}}^{\bar{x}} i d i=\frac{\left(\bar{x}^{2}-\underline{x}^{2}\right)}{2}$, cannot be larger than $\frac{1}{2}$.

${ }^{41}$ An alternative interpretation of $f(\underline{x})$ is the dispersion of bias within the group of informed users. Then, when the least biased informed user is not very biased, the manager disseminates to users with more aligned preferences.
} 
achieve the desired sensitivity of the average action. Not surprisingly, the fraction of informed users is increasing in $k$, i.e., the more biased the manager, the more she restricts the access to information.

\section{Concluding Remarks}

The results in this paper call into doubt the commonly held belief that firms should grant unrestricted access to information to all interested parties. I find that, when firms can selectively disseminate information, they may gather more precise information. Ironically, when the incentive conflict between managers and users is sufficiently severe, leveling the playing field leads to less aggregate information in the marketplace.

A key application of my model is information dissemination by managers to analysts. However, the model can be applied to other settings in which one party can gather information that is relevant for the decisions of others (for example, a CFO who implements an information system that provides decision-facilitating information to divisional managers).

The paper assumes truthful communication of information, which seems to fit well the stringent reporting environment after Dodd Frank and the internal control requirements after Sarbanes-Oxley Section 404. It offers potential for future

research of related questions. For example, I assume that the manager's choice of precision is observable. This may not always be the case. If the payoff function of the manager is commonly known, then the users can conjecture the manager's choice in which case the equilibrium described in this paper persist (although, perhaps, not unique anymore). However, if the players' preference misalignment 
is unknown, then the users will evaluate the expected state of nature using a conjecture of the manager's precision that is based on expectation of the preference misalignment. Future work may analyze several questions that arise. For example, will the manager find it optimal to disclose her precision choice as a signal of her preference misalignment and, if so, how will this affect the equilibrium?

Moreover, this paper assumes that the users take their actions simultaneously. As a result, the uninformed users cannot update their beliefs about the state of nature by observing the informed users' actions. Future work can introduce noise into the users' actions and examine the effects of herding between users. 


\section{References}

[1] Agrawal, A., Chadha, S., Chen, M.A., 2006. "Who Is Afraid of Reg FD? The Behavior and Performance of Sell-Side Analysts Following the SEC's Fair Disclosure Rules," Journal of Business 79(6), 2811-2834.

[2] Arya, A., J. Glover, K. Sivaramakrishnan, 1997. "Commitment issues in budgeting," Journal of Accounting Research 35, 273-278.

[3] Arya, A., J. Glover, B. Mittendorf, and G. Narayanamoorthy, 2005. "Unintended Consequences of Regulating Disclosures: The Case of Regulation Fair Disclosure," Journal of Accounting and Public Policy 24 (3), 243-252.

[4] Aumann, R. J., and M. B. Maschler, 1995. "Repeated Games with Incomplete Information," Cambridge, MA: MIT Press.

[5] Baiman, S., 1975. "The evaluation and choice of internal information systems within a multi-person world," Journal of Accounting Research 13, 1-15.

[6] Beyer, A., and I. Guttman, 2011. "The Effect of Trading Volume on Analysts' Forecast Bias," The Accounting Review 86(2).

[7] Brown, L. D., R. Hagerman, P. Griffin, and M. Zmijewski, 1987. "Security analyst superiority relative to univariate time-series models in forecasting quarterly earnings," Journal of Accounting and Economics 9, 61-87.

[8] Brown, L. D., and M.S. Rozeff, 1978. "The superiority of analyst forecasts as measures of expectations: Evidence from earnings," Journal of Finance 33, $1-16$. 
[9] Che, Y., and N. Kartik, 2009. "Opinions as Incentives," Journal of Political Economy 117 (5), 815-860

[10] Chen, Q., T. R. Lewis and Y. Zhang, 2013. "Publicity vs. Clarity When Public Information Serves a Coordination Role," working paper

[11] Conroy, R., and R. Harris, 1987. "Consensus forecasts of corporate earnings: analysts' forecasts and time series methods," Management Science 33, 725-738.

[12] Demski, J., and G. A. Feltham, 1994. "Market response to ÂÉfinancial reports," Journal of Accounting and Economics, 17, 3-40.

[13] Fischer, P., and P. Stocken, 2010. "Analyst Information Acquisition and Communication," The Accounting Review 85(6), 1985-2009.

[14] Francis, J., Nanda, D., Wang, X., 2006. "Re-examining the effects of Regulation Fair Disclosure using foreign listed firms to control for concurrent shocks," Journal of Accounting and Economics 41 (3), 271-292.

[15] Fried, D., and D. Givoly, 1982. "Financial analysts' forecasts of earnings: A better surrogate for market expectations," Journal of Accounting and Economics 4, 85-107.

[16] Gao, P. and P. Liang, 2013. "Informational Feedback Effect, Adverse Selection, and the Optimal Disclosure Policy," Journal of Accounting Research, conditionally accepted.

[17] Givoly, D., and J. Lakonishok, 1984. "The quality of analysts' forecasts of earnings," Financial Analysts Journal 40, 40-47. 
[18] Groysberg, B., P. Healy, and D. Maber, 2011. "What drives sell-side analyst compensation at high-status investment banks?" Journal of Accounting Research 49(4), 969-1000.

[19] Harsanyi, J., 1955. "Cardinal Welfare, Individualistic Ethics, and Interpersonal Comparisons of Utility," Journal of Political Economy 63(4), 309-321.

[20] Heflin, F., K. R. Subramanyam, and Y. Zhang, 2003. "Regulation FD and the financial information environment: Early evidence," The Accounting Review 78, 1-37.

[21] Herrmann, D., Hope, O., Thomas, W., 2008. "International diversification and forecast optimism: the effects of Reg FD," Accounting Horizons 22 (2), 179-197.

[22] Hughes, J. and S. Pae, 2004. "Voluntary Disclosure of Precision Information," Journal of Accounting and Economics 37(2), 261-289.

[23] Jorgensen, B., J. Li and N. Melumad, 2013. "Why do Firms Gravitate to Selective Disclosure?," working paper

[24] Kamenica, E., and M. Gentzkow, 2011. "Bayesian Persuasion," American Economic Review 101, 2590-2615.

[25] Kim, O., and R.E. Verrecchia, 1991. "Market reaction to anticipated announcements," Journal of Financial Economics 30, 273-309.

[26] Kross, W., B. Ro, and D. Schroeder, 1990. "Earnings expectations: Analysts' information advantage," The Accounting Review 65, 461-476. 
[27] McCloskey, D., and A. Klamer, 1995. "One Quarter of GDP Is Persuasion," American Economic Review 85(2), 191-95.

[28] McNichols, M., and B. Trueman, 1994. "Public disclosure, private information collection, and short-term trading," Journal of Accounting and Economics 17, 69-94.

[29] Melumad, N., and T. Shibano, 1991. "Communication in Settings with No Transfers," The RAND Journal of Economics 22(2), 173-198.

[30] O'Brien, P. C., 1988. "Analysts' forecasts as earnings expectations," Journal of Accounting and Economics 10, 53-83.

[31] Pae, S., 1999. "Acquisition and discretionary disclosure of private information and its implications for firms' productive activities," Journal of Accounting Research 37, 465-474.

[32] Penno, M.C., 1996. "Unobservable precision choices in financial reporting," Journal of Accounting Research 34, 141-149.

[33] Titman, S. and B. Trueman, 1986. "Information Quality and the Valuation of New Issues," Journal of Accounting and Economics (June 1986): 159-72. 


\section{Appendix}

Proof of Proposition 1: Note that $V(x) \equiv V(x, \hat{\pi}(x))$ is single-peaked at $x=k$.

To see this start with $x=k$. By Lemma 1 , the manager implements a perfectly revealing information system so that $\mu_{s, \pi}=\omega, \hat{A}()=.k \omega+(1-k) \mu_{0}$ and

$$
\begin{aligned}
V(k) & =-E_{t=1}\left[\left(k \omega+(1-k) \mu_{0}-k \omega-(1-k) \bar{\omega}\right)^{2}\right] \\
& =-(1-k)^{2}\left(\mu_{0}-\bar{\omega}\right)^{2}
\end{aligned}
$$

Now consider $x=k+\eta_{1}$, where $\eta_{1} \in(0, k)$. By Lemma 1 , the manager implements a perfectly revealing information system so that $\mu_{s, \pi}=\omega, \hat{A}()=.\left(k+\eta_{1}\right) \omega+(1-$ $\left.k-\eta_{1}\right) \mu_{0}$ and

$$
\begin{aligned}
V\left(k+\eta_{1}\right) & =-E_{t=1}\left[\left(\left(k+\eta_{1}\right) \omega+\left(1-k-\eta_{1}\right) \mu_{0}-k \omega-(1-k) \bar{\omega}\right)^{2}\right] \\
& =-E_{t=1}\left[\left(\eta_{1}\left(\omega-\mu_{0}\right)+(1-k)\left(\mu_{0}-\bar{\omega}\right)\right)^{2}\right] \\
& =-\eta_{1}^{2} E_{t=1}\left[\left(\omega-\mu_{0}\right)^{2}\right]-(1-k)^{2}\left(\mu_{0}-\bar{\omega}\right)^{2} \\
& =-\eta_{1}^{2} \operatorname{Var}(\omega)-(1-k)^{2}\left(\mu_{0}-\bar{\omega}\right)^{2} \\
& <-(1-k)^{2}\left(\mu_{0}-\bar{\omega}\right)^{2} \\
& =V(k) .
\end{aligned}
$$


Similarly, $V\left(k-\eta_{1}\right)<V(k)$. Now consider $x=k+\eta_{2}$, where $\eta_{2} \geq k$. By Lemma 1 , the manager does not implement an information system so $\hat{A}()=.\mu_{0}$ and

$$
\begin{aligned}
V\left(k+\eta_{2}\right) & =-E_{t=1}\left[\left(\mu_{0}-k \omega-(1-k) \bar{\omega}\right)^{2}\right] \\
& =-E_{t=1}\left[\left(-k\left(\omega-\mu_{0}\right)+(1-k)\left(\mu_{0}-\bar{\omega}\right)\right)^{2}\right] \\
& =-k^{2} E_{t=1}\left[\left(\omega-\mu_{0}\right)^{2}\right]-(1-k)^{2}\left(\mu_{0}-\bar{\omega}\right)^{2} \\
& =-k^{2} \operatorname{Var}(\omega)-(1-k)^{2}\left(\mu_{0}-\bar{\omega}\right)^{2} \\
& <-\eta_{1}^{2} \operatorname{Var}(\omega)-(1-k)^{2}\left(\mu_{0}-\bar{\omega}\right)^{2} \\
& =V\left(k+\eta_{1}\right) \\
& <V(k) .
\end{aligned}
$$

It follows that the manager will implement a perfectly revealing information system and reveal the signal to a fraction $k$ of users.

Proof of Lemma 2: Differentiating the manager's objective in (8) and using (7) with respect to $\beta$,

$$
\begin{aligned}
\frac{\partial V(x, \beta)}{\partial \beta}-\frac{\partial C(c, \beta)}{\partial \beta} & =-\frac{\partial}{\partial \beta} E_{t=1}\left[\left(x(\varepsilon+\delta) \frac{\beta}{\alpha+\beta}-k \varepsilon\right)^{2}\right]-\frac{\partial C(c, \beta)}{\partial \beta} \\
& =\frac{\partial}{\partial \beta}\left(\frac{x(2 k-x) \beta}{\alpha(\alpha+\beta)}-\frac{k^{2}}{\alpha}\right)-\frac{\partial C(c, \beta)}{\partial \beta} \\
& =\frac{(2 k-x) x}{(\alpha+\beta)^{2}}-\frac{\partial C(c, \beta)}{\partial \beta} .
\end{aligned}
$$

If $k \leq \frac{x}{2}$, then (15) is negative for any precision in the domain, and the manager does not implement an information system (equivalently, sets $\beta=0$ ). If $k>\frac{x}{2}$, 
then there exist $\beta^{c}(x)$ that satisfies FOC:

$$
\frac{(2 k-x) x}{(\alpha+\beta)^{2}}-\left.\frac{\partial C(c, \beta)}{\partial \beta}\right|_{\beta=\beta^{c}(x)}=0
$$

Applying the Implicit Function Theorem,

$$
\begin{aligned}
& \frac{\partial \beta^{c}(x)}{\partial c}=-\frac{\frac{\partial^{2} C(c, \beta)}{\partial \beta \partial c}}{\frac{2 x(2 k-x)}{(\alpha+\beta)^{3}}+\frac{\partial^{2} C(c, \beta)}{\partial \beta^{2}}}<0 \\
& \frac{\partial \beta^{c}(x)}{\partial \alpha}=-\frac{\frac{2(2 k-x) x}{(\alpha+\beta)^{3}}}{\frac{2 x(2 k-x)}{(\alpha+\beta)^{3}}+\frac{\partial^{2} C(c, \beta)}{\partial \beta^{2}}}<0 \\
& \frac{\partial \beta^{c}(x)}{\partial k}=\frac{\frac{2 x}{(\alpha+\beta)^{2}}}{\frac{2 x(2 k-x)}{(\alpha+\beta)^{3}}+\frac{\partial^{2} C(c, \beta)}{\partial \beta^{2}}}>0,
\end{aligned}
$$

because $k>\frac{x}{2}$. Lastly, note that that $\beta^{c}(x)$ is single peaked at $x=k$ because, by the Implicit Function Theorem,

$$
\frac{\partial \beta^{c}(x)}{\partial x}=-\frac{\frac{2(x-k)}{(\alpha+\beta)^{2}}}{\frac{2 x(2 k-x)}{(\alpha+\beta)^{3}}+\frac{\partial^{2} C(c, \beta)}{\partial \beta^{2}}}\left\{\begin{array}{l}
>0 \text { if } x<k, \\
=0 \text { if } x=k, \\
<0 \text { if } x>k .
\end{array}\right.
$$


Proof of Proposition 3: Differentiating the manager's objective in (9),

$$
\begin{aligned}
\frac{\partial V(x, \beta)}{\partial \beta}-\frac{\partial C(c, \beta)}{\partial \beta} & =-\frac{\partial}{\partial \beta} E_{t=1}\left[\left(x(\varepsilon+\delta) \frac{\beta}{\alpha+\beta}-k \varepsilon\right)^{2}\right]-\frac{\partial C(c, \beta)}{\partial \beta} \\
& =\frac{\partial}{\partial \beta}\left(\frac{x(2 k-x) \beta}{\alpha(\alpha+\beta)}-\frac{k^{2}}{\alpha}\right)-\frac{\partial C(c, \beta)}{\partial \beta} \\
= & \frac{x(2 k-x)}{(\alpha+\beta)^{2}}-\frac{\partial C(c, \beta)}{\partial \beta} \\
\frac{\partial V(x, \beta)}{\partial x}-\frac{\partial C(c, \beta)}{\partial x} & =-\frac{\partial}{\partial x} E_{t=1}\left[\left(x(\varepsilon+\delta) \frac{\beta}{\alpha+\beta}-k \varepsilon\right)^{2}\right] \\
& =\frac{\partial}{\partial x}\left(\frac{x(2 k-x) \beta}{\alpha(\alpha+\beta)}-\frac{k^{2}}{\alpha}\right) \\
& =\frac{2 \beta(k-x)}{\alpha(\alpha+\beta)}
\end{aligned}
$$

The critical points satisfying (17) and (18) simultaneously are

$$
(x, \beta) \in\{(k, \tilde{\beta}),(0,0),(2 k, 0)\}
$$

where $\tilde{\beta}$ satisfies $\frac{k^{2}}{(\alpha+\beta)^{2}}-\left.\frac{\partial C(c, \beta)}{\partial \beta}\right|_{\beta=\tilde{\beta}}=0$. To verify SOC, I examine the Hessian:

$$
H=\left[\begin{array}{cc}
-\frac{2 \beta}{\alpha(\alpha+\beta)} & \frac{2(k-x)}{(\alpha+\beta)^{2}} \\
\frac{2(k-x)}{(\alpha+\beta)^{2}} & -\frac{2(2 k-x) x}{(\alpha+\beta)^{3}}-\frac{\partial^{2} C(c, \beta)}{\partial \beta^{2}}
\end{array}\right]
$$


At $(\tilde{x}, 0)$, where $\tilde{x} \in\{0,2 k\}$, the Hessian is

$$
H=\left[\begin{array}{cc}
0 & \frac{2(k-\tilde{x})}{\alpha^{2}} \\
\frac{2(k-\tilde{x})}{\alpha^{2}} & -\frac{2(2 k-\tilde{x}) \tilde{x}}{(\alpha+\beta)^{3}}
\end{array}\right]
$$

so $\left|H_{1}\right|=0$ and $\left|H_{2}\right|<0$. However, at $(k, \tilde{\beta})$, the Hessian is

$$
H=\left[\begin{array}{cc}
-\frac{2 \tilde{\beta}}{\alpha(\alpha+\tilde{\beta})} & 0 \\
0 & -\frac{2 k^{2}}{(\alpha+\tilde{\beta})^{3}}-\frac{\partial^{2} C(c, \tilde{\beta})}{\partial \beta^{2}}
\end{array}\right]
$$

so $\left|H_{1}\right|<0$ and $\left|H_{2}\right|>0$. Therefore $\left(x_{U D}^{c}, \beta_{U D}^{c}\right)=(k, \tilde{\beta})$. Next note that $\tilde{\beta}=$ $\beta^{c}(x=k)$. Hence, if the manager can choose the fraction of informed users, she will set $x_{U D}^{c}=k$ and $\beta_{U D}^{c} \equiv \beta^{c}(x=k)$.

Proof of Proposition 4: The result for the manager follows by revealed preference. To evaluate the users' preferences, note that a user who observes a signal incurs in expectation the posterior variance and his payoff is increasing in the signal 
precision $\beta$ :

$$
\begin{aligned}
\frac{\partial}{\partial \beta} E_{t=1}[u(\hat{a}(s, \beta), \omega)] & =-\frac{\partial}{\partial \beta} E_{t=1}\left[\left(\mu_{0}+\frac{\beta(\varepsilon+\delta)}{\alpha+\beta}-\omega\right)^{2}\right] \\
& =-\frac{\partial}{\partial \beta} E_{t=1}\left[\left(\frac{\beta \delta}{\alpha+\beta}-\frac{\alpha \varepsilon}{\alpha+\beta}\right)^{2}\right] \\
& =-\frac{\partial}{\partial \beta}\left(\frac{\beta^{2}}{(\alpha+\beta)^{2}} E_{t=1}\left[\delta^{2}\right]-\frac{\alpha^{2}}{(\alpha+\beta)^{2}} E_{t=1}\left[\varepsilon^{2}\right]\right) \\
& =-\frac{\partial}{\partial \beta}\left(\frac{1}{\alpha+\beta}\right) \\
& =\frac{1}{(\alpha+\beta)^{2}}>0 .
\end{aligned}
$$

It immediately follows that

$$
E_{t=1}[u(\hat{a}(s, \beta), \omega)] \geq E_{t=1}[u(\hat{a}(\emptyset), \omega)], \forall \beta \geq 0 .
$$

Case $k \leq \frac{1}{2}$ : By Lemma 2 and Proposition 3, the manager implements an information system only under UD. The uninformed users are indifferent, because they do not observe a signal under either of the regimes. By (20), the informed users are better off under UD.

Case $k>\frac{1}{2}$ : By Lemma 2 and Proposition 3, the manager implements an information system under both regimes. By (20), the uninformed users are strictly better off under MD (because they observe a signal, while under UD they do not). By Lemma 2 and (19), the informed users strictly prefer UD.

Proof of Corollary 2: Note that the information gain is increasing in the preci- 
sion, because

$$
\frac{\partial}{\partial \beta}\left(E_{t=1}[u(\hat{a}(s, \beta), \omega)]-E_{t=1}[u(\hat{a}(\emptyset), \omega)]\right)=\frac{\partial}{\partial \beta} E_{t=1}[u(\hat{a}(s, \beta), \omega)]>0
$$

by (19). Then, by Lemma $2, \phi^{c}(x) \equiv E_{t=1}\left[u\left(\hat{a}\left(s, \beta^{c}(x)\right), \omega\right)\right]-E_{t=1}[u(\hat{a}(\emptyset), \omega)]$ is decreasing in $\Delta x \equiv|x-k|$.

Proof of Proposition 5: Consider the differential of the welfare terms under the two regimes:

$$
\Delta W \equiv W_{U D}^{c}-W_{M D}^{c}=k \phi_{U D}^{c}-\phi_{M D}^{c}
$$

The sign of $\Delta W$ is nontrivial (because $k<1$ by assumption but $\phi_{U D}^{c}>\phi_{M D}^{c}$ by Corollary 2). Note that $\lim _{c \rightarrow 0} \beta_{M D}^{c}=\lim _{c \rightarrow 0} \beta_{U D}^{c} \rightarrow \infty$. Note that for $j=$ $U D, M D$

$$
\begin{aligned}
\phi_{j}^{c} & =E_{t=1}\left[u\left(\hat{a}\left(s, \beta_{j}^{c}\right), \omega\right) \mid s, \beta_{j}^{c}\right]-E_{t=1}[u(\hat{a}(\emptyset), \omega)] \\
& =-\frac{1}{\alpha+\beta_{j}^{c}}+\frac{1}{\alpha} \\
& =\frac{\beta_{j}^{c}}{\alpha\left(\alpha+\beta_{j}^{c}\right)} .
\end{aligned}
$$

It follows that $\lim _{c \rightarrow 0} \phi_{M D}^{c}=\lim _{c \rightarrow 0} \phi_{U D}^{c}=\frac{1}{\alpha}$ and hence

$$
\lim _{c \rightarrow 0} \Delta W \propto k-1<0
$$

Further, $\lim _{c \rightarrow \infty} \beta_{M D}^{c}=\lim _{c \rightarrow \infty} \beta_{U D}^{c}=0$, so $\lim _{c \rightarrow \infty} \phi_{M D}^{c}=\lim _{c \rightarrow \infty} \phi_{U D}^{c}=0$ and 
hence

$$
\lim _{c \rightarrow \infty} \Delta W=0 .
$$

However, $\phi_{M D}^{c}$ reaches zero weakly faster than $k \phi_{U D}^{c}$ if $k \leq \frac{1}{2}(\sqrt{5}-1)$. To see why consider:

$$
\Phi \equiv \frac{k \phi_{U D}^{c}}{\phi_{M D}^{c}}=\frac{\frac{k \beta_{U D}^{c}}{\alpha\left(\alpha+\beta_{U D}^{c}\right)}}{\frac{\beta_{M D}^{c}}{\alpha\left(\alpha+\beta_{M D}^{c}\right)}}
$$

Note that, if $C(c, \beta)=\frac{c \beta^{2}}{2}$, then $\beta_{U D}^{c}$ and $\beta_{M D}^{c}$ satisfy

$$
\begin{aligned}
& \beta_{U D}^{c}=\frac{k^{2}}{c\left(\alpha+\beta_{U D}^{c}\right)^{2}}, \\
& \beta_{M D}^{c}=\frac{2 k-1}{c\left(\alpha+\beta_{M D}^{c}\right)^{2}} .
\end{aligned}
$$

and hence

$$
\Phi=\frac{\frac{k^{3}}{\alpha c\left(\alpha+\beta_{U D}^{c}\right)^{3}}}{\frac{2 k-1}{\alpha c\left(\alpha+\beta_{M D}^{c}\right)^{3}}}=\frac{k^{3}\left(\alpha+\beta_{M D}^{c}\right)^{3}}{(2 k-1)\left(\alpha+\beta_{U D}^{c}\right)^{3}} .
$$

Now note that

$$
\lim _{c \rightarrow \infty} \Phi=\frac{k^{3}}{2 k-1} \geq 1
$$

if $k \leq \frac{1}{2}(\sqrt{5}-1)$. In other words, $\phi_{M D}^{c}$ reaches zero weakly faster than $k \phi_{U D}^{c}$ if $k \leq \tilde{k} \equiv \frac{1}{2}(\sqrt{5}-1) \in\left(\frac{1}{2}, 1\right)$. It follows that for $c$ sufficiently high and $k \leq \tilde{k}$, $\Delta W \geq 0$. 
Proof of Lemma 3: Let

$$
\Pi^{c}(x) \equiv \lambda\left[V\left(x, \beta^{c}(x)\right)-C\left(c, \beta^{c}(x)\right)\right]+W^{c}(x)
$$

denote the objective function of the planner with costly implementation, where

$$
\beta^{c}(x)=\arg \max _{\beta} V(x, \beta)-C(c, \beta) .
$$

The optimal $\hat{x}_{R D}^{c}$ satisfies $\left.\frac{d \Pi^{c}(x)}{d x}\right|_{x=\hat{x}_{R D}^{c}}=0$. Differentiating with respect to $x$,

$$
\begin{aligned}
\frac{d \Pi^{c}(x)}{d x}= & \lambda \underbrace{\left.\left(\frac{\partial V(x, \beta)}{\partial \beta}-\frac{\partial C(c, \beta)}{\partial \beta}\right)\right|_{\beta=\beta^{c}(x)} \frac{d \beta^{c}(x)}{d x}}_{=0 \text { by FOC }} \\
& +\lambda \frac{\partial V\left(x, \beta^{c}(.)\right)}{\partial x}+\phi^{c}(x)+x \frac{d \phi^{c}(x)}{d x} \\
= & \frac{1}{\alpha+\beta^{c}(x)}\left[\frac{\beta^{c}(x)(1+2 \lambda(k-x))}{\alpha}+\frac{x}{\left(\alpha+\beta^{c}(x)\right)} \frac{\partial \beta^{c}(x)}{\partial x}\right] .
\end{aligned}
$$

Let $g(x) \equiv \frac{\beta^{c}(x)(1+2 \lambda(k-x))}{\alpha}+\frac{x}{\left(\alpha+\beta^{c}(x)\right)} \frac{\partial \beta^{c}(x)}{\partial x}$. Given that $\frac{1}{\alpha+\beta^{c}(x)}>0, \hat{x}_{R D}^{c}$ satisfies $\left.g(x)\right|_{x=\hat{x}_{R D}^{c}}=0$. Next observe that, if $C(c, \beta)=\frac{c \beta^{2}}{2}$, then $\beta^{c}(x)$ satisfies

$$
\frac{(2 k-x) x}{\left(\alpha+\beta^{c}(x)\right)^{2}}-c \beta^{c}(x)=0
$$

and applying the Implicit Function Theorem,

$$
\frac{d \beta^{c}(x)}{d x}=\frac{\frac{2(k-x)}{\left(\alpha+\beta^{c}(x)\right)^{2}}}{\frac{2 x(2 k-x)}{\left(\alpha+\beta^{c}(x)\right)^{3}}+c}=\frac{(k-x)\left(\alpha+\beta^{c}(x)\right)}{(2 k-x) x} .
$$


Substituting,

$$
g(x)=\frac{\beta^{c}(x)(1+2 \lambda(k-x))}{\alpha}+\frac{k-x}{2 k-x} .
$$

Note that $\hat{x}_{R D}^{c} \geq k$ because

$$
g(x=k)=\frac{\beta^{c}(x=k)}{\alpha} \geq 0 .
$$

Observe that $\hat{x}_{R D}^{c}<2 k$ (to satisfy the implementation constraint), because by Lemma 2 , if $x \geq 2 k$, then $\beta^{c}(x)=0$. If $k \leq \frac{1}{2}$, then it follows directly that $\hat{x}_{R D}^{c}<1$ (because $2 k<1$ ). However, if $k>\frac{1}{2}$, then $\hat{x}_{R D}^{c}<1$ only if $c$ sufficiently high. To see why first consider $c=0$.

$$
\lim _{c \rightarrow 0} g(x=1)=\frac{\lim _{c \rightarrow 0} \beta^{c}(x=1)(1+2 \lambda(k-1))}{\alpha}+\frac{k-1}{2 k-1}>0
$$

because when $k>\frac{1}{2}$ then $1+2 \lambda(k-1)>1+2 \lambda\left(\frac{1}{2}-1\right)=1-\lambda \geq 0$ (because $\lambda \leq 1$ by assumption) and $\lim _{c \rightarrow 0} \beta^{c}(x=1) \rightarrow \infty$. In other words, if $c=0$ and $k>\frac{1}{2}$, the planner sets $x_{R D}^{c}=1$. However,

$$
\begin{aligned}
g(x=1) & =\frac{\beta^{c}(x=1)(1+2 \lambda(k-1))}{\alpha}+\frac{k-1}{2 k-1} \\
& \propto \beta^{c}(x=1)(2 k-1)(1+2 \lambda(k-1))+(k-1) \alpha<0,
\end{aligned}
$$

if $\beta^{c}(x=1)$ is sufficiently low, which by Lemma 2 occurs when $c$ is sufficiently high (recall that $\lim _{c \rightarrow \infty} \beta^{c}(x=1)=0$ ). It follows that $x_{R D}^{c}<1$ if $c$ is sufficiently high for any $k$.

To show the comparative statics of $x_{R D}^{c}$ with respect to $c$, I apply the Implicit 
Function Theorem:

$$
\begin{aligned}
\frac{\partial x_{R D}^{c}}{\partial c} & =-\frac{\frac{\left(1+2 \lambda\left(k-x_{R D}^{c}\right)\right)}{\alpha} \frac{\partial \beta^{c}\left(x=x_{R D}^{c}\right)}{\partial c}}{\frac{-\alpha k+\left(x_{R D}^{c}-2 k\right)^{2}\left(-2 \lambda \beta^{c}\left(x=x_{R D}^{c}\right)+\left(1+2 \lambda\left(k-x_{R D}^{c}\right) \frac{\partial \beta^{c}\left(x=x_{R D}^{c}\right)}{\partial x}\right)\right.}{\alpha\left(x_{R D}^{c}-2 k\right)^{2}}} \\
& \propto \frac{1+2 \lambda\left(k-x_{R D}^{c}\right)}{-\alpha k+\left(x_{R D}^{c}-2 k\right)^{2}\left(-2 \lambda \beta^{c}\left(x=x_{R D}^{c}\right)+\left(1+2 \lambda\left(k-x_{R D}^{c}\right)\right) \frac{\partial \beta^{c}\left(x=x_{R D}^{c}\right)}{\partial x}\right)}<0
\end{aligned}
$$

because

(i) $\alpha \geq 0$ by assumption and $\left(x_{R D}^{c}-2 k\right)^{2}>0$;

(ii) $\frac{\partial \beta^{c}\left(x=x_{R D}^{c}\right)}{\partial c}<0$ by Lemma 2 ;

(ii) $\frac{\partial \beta^{c}\left(x=x_{R D}^{c}\right)}{\partial x}=\frac{\left(k-x_{R D}^{c}\right)\left(\alpha+\beta^{c}\left(x=x_{R D}^{c}\right)\right)}{\left(2 k-x_{R D}^{c}\right) x_{R D}^{c}} \propto \frac{\left(k-x_{R D}^{c}\right)}{\left(2 k-x_{R D}^{c}\right)}<0$, because $x_{R D}^{c} \in(k, 2 k)$;

(iii) $1+2 \lambda\left(k-x_{R D}^{c}\right)>0$ if $\lambda \in[0,1]$. To see this, recall from the preceding discussion in the proof that $x_{R D}^{c}<\operatorname{Min}\{2 k, 1\}$. Therefore, if $k \leq \frac{1}{2}$, then $\operatorname{Min}\{2 k, 1\}=2 k$ and $1+2 \lambda\left(k-x_{R D}^{c}\right)>1+2 \lambda(k-2 k)=1-2 \lambda k>$ $1-2 k>0$ (because $\lambda \leq 1$ by assumption). If $k>\frac{1}{2}$, then $\operatorname{Min}\{2 k, 1\}=1$ and $1+2 \lambda\left(k-x_{R D}^{c}\right)>1+2 \lambda(k-1)>1+2 \lambda\left(\frac{1}{2}-1\right)=1-\lambda \geq 0$ (because $\lambda \leq 1$ by assumption)

Proof of Proposition 6: The manager's expected payoff is:

$$
\begin{aligned}
V^{H}(\underline{x}, \bar{x}, \pi) & =-E_{t=1}\left[\left(\frac{\mu_{0}+\underline{\omega}}{2}+\frac{\left(\bar{x}^{2}-\underline{x}^{2}\right)}{2}\left(\mu_{s, \pi}-\mu_{0}\right)-k \omega-(1-k) \bar{\omega}\right)^{2}\right] \\
& =-E_{t=1}\left[\left(\frac{\left(\bar{x}^{2}-\underline{x}^{2}\right)}{2}\left(\mu_{s, \pi}-\mu_{0}\right)-k \omega\right)^{2}\right]-\left(\frac{\mu_{0}+\underline{\omega}}{2}-(1-k) \bar{\omega}\right)^{2}
\end{aligned}
$$


Let define $D \equiv \bar{x}^{2}-\underline{x}^{2}$ and rewrite the expected payoff of the manager as:

$$
V^{H}(D, \pi)=-E_{t=1}\left[\left(\frac{D}{2}\left(\mu_{s, \pi}-\mu_{0}\right)-k \omega\right)^{2}\right]-\left(\frac{\mu_{0}+\underline{\omega}}{2}-(1-k) \bar{\omega}\right)^{2}
$$

Step 1: Suppose $D$ is exogenous and the manager chooses

$$
\pi^{H}(D) \equiv \arg \max _{\pi} V^{H}(D, \pi)
$$

Following the logic of the proofs of Lemma 1 and Corollary 1 it is straightforward to show that if $k>\frac{D}{4}\left(k \leq \frac{D}{4}\right)$ the manager implements a perfectly informative system (does not implement an information system).

Step 2: Following the logic of the proof of Proposition 1, it is straightforward to show that $V^{H}(D) \equiv V^{H}\left(D, \pi^{H}(D)\right)$ is single peaked at $D=2 k$.

Step 3: Recall that $D \equiv \bar{x}^{2}-\underline{x}^{2}$. The manager wants to disseminate the signal to a fraction of informed users that satisfies $\bar{x}^{2}-\underline{x}^{2}=2 k$. Let define $f(\underline{x}) \equiv \sqrt{2 k+(\underline{x})^{2}}-\underline{x}$. Then, the partition of users to whom the manager wants to reveal the signal can be presented as $[\underline{x}, \underline{x}+f(\underline{x})]$. Consider $k<\frac{1}{2}$ and $\underline{x}^{o}=0$. Then, $\bar{x}^{o}=\sqrt{2 k+\left(\underline{x}^{o}\right)^{2}}=\sqrt{2 k}<1$ achieves maximum payoff to the manager. Next, consider $k<\frac{1}{2}$ and $\underline{x}^{o o}=\bar{x}^{o}+\xi=\xi$ with $\xi>0$ arbitrarily small. There exist $\bar{x}^{o o}=\sqrt{2 k+\left(\underline{x}^{o o}\right)^{2}}=\sqrt{2 k+\xi^{2}}$ that achieves the same maximum payoff to the manager. Given that $\bar{x} \leq 1$ by assumption, the pair $(\underline{x}, \bar{x})$ with largest $\bar{x}$ that achieves this payoff is $(\sqrt{1-2 k}, 1)$. Note, that if $k<\frac{1}{2}$, then $\sqrt{1-2 k}>0$. Next, consider $k=\frac{1}{2}$. Then, the only pair $(\underline{x}, \bar{x})$ that achieves this payoff is $(0,1)$. Now note that, if $k>\frac{1}{2}$, then $\sqrt{2 k+\underline{x}^{2}}>1$ for any $\underline{x} \in[0,1]$. Given that $0 \leq \underline{x} \leq \bar{x} \leq 1$ by assumption, the manager sets $\underline{x}=0$ and $\bar{x}=1$.

Step 4: Lastly, note that, since $0<k<1$ and $0 \leq \underline{x} \leq 1$ by assumption, then 
$\frac{\partial f(.)}{\partial \underline{x}}=\frac{\underline{x}}{\sqrt{2 k+\underline{x}^{2}}}-1<1-1=0$ and $\frac{\partial f(.)}{\partial k}=\frac{\underline{1}}{\sqrt{2 k+\underline{x}^{2}}}>0$. 